Vietnam Journal of Mechanics, VAST, Vol. 42, No. 3 (2020), pp. $209-237$

DOI: https://doi.org/10.15625/0866-7136/15336

Dedicated to Professor J.N. Reddy on the Occasion of His $75^{\text {th }}$ Birthday

\title{
THE METHOD OF FINITE SPHERES IN ACOUSTIC WAVE PROPAGATION THROUGH NONHOMOGENEOUS MEDIA: INF-SUP STABILITY CONDITIONS
}

\author{
Williams L. Nicomedes ${ }^{1, *}$, Klaus-Jürgen Bathe ${ }^{2}$, Fernando J. S. Moreira ${ }^{3}$, Renato C. Mesquita ${ }^{4}$ \\ ${ }^{1}$ Graduate Program in Electrical Engineering, Federal University of Minas Gerais, Belo Horizonte MG, Brazil \\ ${ }^{2}$ Department of Mechanical Engineering, Massachusetts Institute of Technology, Cambridge MA, USA \\ ${ }^{3}$ Department of Electronics Engineering, Federal University of Minas Gerais, Belo Horizonte MG, Brazil \\ ${ }^{4}$ Department of Electrical Engineering, Federal University of Minas Gerais, Belo Horizonte MG, Brazil \\ *E-mail: wnicomedes@eng-ele.grad.ufmg.br
}

Received: 26 July 2020 / Published online: 27 September 2020

\begin{abstract}
When the method of finite spheres is used for the solution of time-harmonic acoustic wave propagation problems in nonhomogeneous media, a mixed (or saddle-point) formulation is obtained in which the unknowns are the pressure fields and the Lagrange multiplier fields defined at the interfaces between the regions with distinct material properties. Then certain inf-sup conditions must be satisfied by the discretized spaces in order for the finite-dimensional problems to be well-posed. We discuss in this paper the analysis and use of these conditions. Since the conditions involve norms of functionals in fractional Sobolev spaces, we derive 'stronger' conditions that are simpler in form. These new conditions pave the way for the inf-sup testing, a tool for assessing the stability of the discretized problems.
\end{abstract}

Keywords: acoustic waves, finite elements, finite spheres, inf-sup conditions, meshfree methods.

\section{INTRODUCTION}

\subsection{Overview}

The method of finite spheres (MFS) is a meshfree method [1], such as the smoothed particle hydrodynamics (SPH) [2], the element-free Galerkin (EFG) [3], and the meshless local Petrov-Galerkin methods [4]. While each of these procedures have been used differently [5], the basic characteristic shared by all of them is the complete absence of meshes, as those employed in the traditional finite element method [6].

The MFS is a truly meshfree method (in the sense that the numerical integrations are carried out locally on the subdomains) and leads to sparse linear systems of algebraic equations. The method has also been used in the AMORE scheme of analysis [7] and it is the basis for the development of the 'overlapping finite elements' [8]. First proposed as a tool for the analysis of solids, the MFS has also successfully been applied to electromagnetic wave scattering problems [9]. Building on these results, we conducted a study [10] in which we used the MFS to solve time-harmonic acoustic wave propagation problems in nonhomogeneous media [11]. In these solutions, objects of different material properties (density and bulk modulus) are considered in a homogeneous host medium. The discontinuity of material properties across the interfaces between the objects and the host medium leads to jumps in the gradients of the pressure field. If a meshfree method is used for the solution of such problems, oscillations in the predicted response are observed unless specially treated. The pressure field is governed by the Helmholtz equation, and we use a Lagrange multiplier field to impose the discontinuity of the gradients in a weak sense. Thus we are led to a two-field mixed formulation [6,12-14] in which we seek 
to solve for a primary field (in this case, the pressure field) and a secondary field (given by the Lagrange multiplier field).

The present paper can be regarded to be a companion paper to [10]. In the following, we show in detail how the weak formulation naturally leads to the use of Lagrange multipliers and the relevant inf-sup conditions. We recast these inf-sup conditions into forms easier to evaluate. In particular, these final inf-sup conditions can be used for a numerical inf-sup test. In a sense, this presentation provides the theoretical foundation for the numerical simulations carried out in [10].

\subsection{Lagrange multiplier fields and dual norms}

In the standard variational formulation of scalar problems with discontinuous gradients (like in acoustic wave propagations in nonhomogeneous media), the Lagrange multiplier field is generally a functional in the space $H^{-1 / 2}(\Gamma)$, the dual space of the fractional Sobolev space $H^{1 / 2}(\Gamma)$, where $\Gamma$ denotes the interface between the media of different material properties [15-17]. Given that the direct evaluation of the $H^{1 / 2}$ norm of functions is an involved task (due to double integrals along $\Gamma$ and singularities in the integrands [16,17]), and that the original inf-sup conditions involve the evaluation of the $H^{-1 / 2}$ dual norm of Lagrange multiplier fields, it is difficult to verify whether these conditions hold true.

There are two approaches to circumvent the difficulties due to the $H^{-1 / 2}$ norms in the inf-sup conditions. In the first, instead of evaluating the inf-sup condition relative to the problem at hand, the focus is directed to the final linear system of algebraic equations [18-22]. Since the dimension of the subspace used to approximate the Lagrange multiplier field must be smaller than the dimension of the subspace used to approximate the pressure field [23], the idea is to obtain an upper limit on the dimension of the first and ensure that the number of Lagrange multiplier constraint equations (in the linear system) remains smaller than this upper limit [6]. The authors arrive at an algebraic relation concerning the suitable number of Lagrange multiplier DoF's (degrees of freedom) to be used. However, this algebraic relation is necessary for the well-posedness of the problem solution, but not sufficient (i.e., if the inf-sup condition holds, then this relation is satisfied, but satisfying this relation does not imply that the inf-sup condition holds).

In the second approach we would transform the inf-sup condition (which involves the $H^{-1 / 2}$ dual norm) into a weaker inf-sup condition that does not involve the $H^{-1 / 2}$ norm. Mesh-dependent norms and inequalities are used, so that the $H^{-1 / 2}$ dual norm of the Lagrange multiplier field is usually substituted by some quantity involving the discretization length $h$ (a characteristic of the mesh) [24-27]. However, in some cases the weaker inf-sup condition is necessary (i.e., if the actual inf-sup condition holds, so does the weaker condition), but not sufficient (i.e., satisfying the weaker condition does not imply that the actual condition holds [24]). In other words, the weaker condition can only be used to rule out possible discretization schemes [24]). Once the weaker condition is established, an inf-sup test can be performed [28].

In this work, we propose a third approach. The difficulty presented by the $H^{-1 / 2}$ norm is removed not by using a weaker condition but by using a stronger inf-sup condition. This is achieved by finding new inf-sup conditions which do not involve the $H^{-1 / 2}$ norm and are stronger than the original conditions (which involve the $H^{-1 / 2}$ norm). Essentially, we look for sufficient conditions: If the new conditions hold, then the original conditions also hold true necessarily. Schematically,

$$
\begin{gathered}
\text { new } \\
\text { inf-sup condition }
\end{gathered} \Rightarrow \begin{gathered}
\text { original } \\
\text { inf-sup condition }
\end{gathered} \Rightarrow \begin{gathered}
\text { Wellposedness } \\
\text { of the discrete problem }
\end{gathered}
$$

The key ingredient is the correct use of an auxiliary theorem which allows us to replace $H^{-1 / 2}$ norms by $H^{1}$ norms in certain geometric settings. The resulting new inf-sup conditions are stronger and at the same time easier to deal with than the original conditions. Once we have established the new stronger conditions, the well-posedness of the discrete problems follows from (1). The stability of the discretized problems can finally be assessed by the aforementioned inf-sup test. This test was originally developed and applied to real-valued variational problems and matrices [28-30]. Since the Helmholtz 
problem examined here is complex-valued in nature, we first treat the complex-valued matrices in order to derive from them certain real-valued matrices. We then apply the inf-sup test.

\subsection{Organization of the manuscript}

In Section 2 we introduce the equations to be solved for the problems considered, together with the assumptions made regarding the geometry of the problem. We derive the weak form of the problem formulation in Section 3 and show how the Lagrange multiplier fields arise naturally. In Section 4 we give a brief discussion of the discretization process using the method of finite spheres. The wellposedness of the variational problem depends on two distinct inf-sup conditions, given in Section 5 . We derive in Sections 6 and 7 more tractable inf-sup conditions which can be used in the inf-sup test. In Section 8, we provide a demonstration of the MFS method, followed by the inf-sup testing procedure in Section 9. Finally, we give our concluding remarks.

\section{EQUATIONS OF WAVE PROPAGATION}

In this section we specify the geometrical properties of the problem and state the equations to be solved.

\subsection{Geometry}

The geometrical setting corresponding to our problem is specified in detail in [10]. In $\mathbb{R}^{2}$, let $B(\mathbf{0} ; R)$ be an open ball with radius $R$ and centered at the origin, see Fig. 1 . The boundary of this region is the circle denoted by $\Gamma_{R}$. Within this region, we place a number of objects with distinct characteristic material properties. The regions occupied by these objects are open subsets of $B(\mathbf{0} ; R)$, which are identified by numerical indices, beginning with 1 . (For example, if our problem is characterized by 3 objects immersed in the host medium as in Fig. 1, the regions occupied by them are $\Omega_{1}, \Omega_{2}$ and $\Omega_{3}$.) The host medium is represented by the set difference between $B(\mathbf{0} ; R)$ and the union of the closures of the regions occupied by the objects. The region corresponding to the host medium will always be indexed by a number equal to the number of objects plus 1 (here $\Omega_{4}$ in Fig. 1). We assume the boundaries of all these regions to be Lipschitz continuous curves. Moreover, given any two regions, their boundaries are such that either they do not touch each other (i.e., they lie at a certain distance from each other, as $\partial \Omega_{1}$ and $\partial \Omega_{3}$ in Fig. 1) or, if they do, then their intersection must be a single closed curve (as $\partial \Omega_{1}$ and $\partial \Omega_{2}$ in Fig. 1). We shall focus our attention on sufficiently regular closed curves, described by a finite number of vertices connected together either by straight segments or by arcs. We refer to [10] for examples of boundaries with different geometrical configurations.

Throughout this paper we will refer to the geometry illustrated in Fig. 1, but the procedures presented below can be generalized to any kind of geometry as long as the above-stated assumptions hold. In the geometrical setting depicted in Fig. 1, the boundaries of each region can be represented by the union of closed curves as

$$
\begin{gathered}
\partial \Omega_{1}=\Gamma_{1,2}, \\
\partial \Omega_{2}=\Gamma_{1,2} \cup \Gamma_{2,4}, \\
\partial \Omega_{3}=\Gamma_{3,4}, \\
\partial \Omega_{4}=\Gamma_{2,4} \cup \Gamma_{3,4} \cup \Gamma_{R},
\end{gathered}
$$

where $\Gamma_{1,2} \stackrel{\text { def }}{=} \partial \Omega_{1} \cap \partial \Omega_{2}$ denotes the interface between regions $\Omega_{1}$ and $\Omega_{2}$, and likewise for the other pairs of indices. So in accordance with the assumptions made above, given any two distinct indices $i$ and $j$ taken from the set $\{1,2,3,4\}$, either $\Gamma_{i, j}$ is the empty set (as $\Gamma_{1,3}$ in Fig. 1$)$, or $\Gamma_{i, j}$ is a single closed curve (as $\Gamma_{1,2}$ in Fig. 1). Regions $\Omega_{1}$ and $\Omega_{3}$ are simply-connected, whereas regions $\Omega_{2}$ and $\Omega_{4}$ are not simplyconnected. The boundaries $\partial \Omega_{2}$ and $\partial \Omega_{4}$ are represented by the union of more than one closed curve, according to (2b) and (2d), respectively. The region representing the host medium will, by definition, always be a not simply-connected domain (i.e., it contains holes left by the objects). 


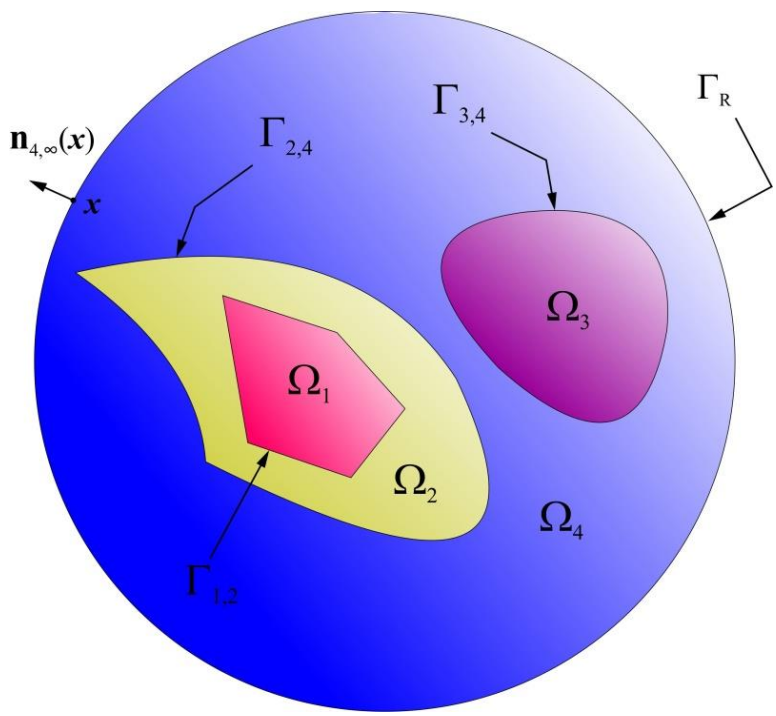

Fig. 1. A geometrical setting with 3 objects, occupying the open regions $\Omega_{1}, \Omega_{2}$, and $\Omega_{3}$. These objects are immersed in the host medium, represented by region $\Omega_{4}$, and given by the set difference between the circle $B(0 ; R)$ and $\bar{\Omega}_{1} \cup \bar{\Omega}_{2} \cup \bar{\Omega}_{3}$, where $\bar{\Omega}_{1}=\Omega_{1} \cup \partial \Omega_{1}$, and so on. This region is shown in blue. The geometry portrayed here is a representative of the class of all geometries amenable to be treated by the methods described in this work. All boundaries $\partial \Omega_{1}, \ldots, \partial \Omega_{4}$ are Lipschitz continuous curves. Regions such as $\Omega_{2}$, which are not simply-connected, can model the cladding of some object, in this case, the object occupying region $\Omega_{1}$

\subsection{The wave equations}

The scattering of acoustic waves considered here refers to an incident (or incoming) pressure wave $p^{i n c}$ propagating in the host medium (represented by a function defined within $B(\mathbf{0} ; R)$ and along its boundary $\Gamma_{R}$ ), which is perturbed by the material objects. Based on our setting, the wave equations to be solved within each region are [11]: For $r=1, \ldots, 4$, find $p_{r}: \bar{\Omega}_{r} \rightarrow \mathbb{C}$ such that for any $x \in \Omega_{r}$,

$$
\boldsymbol{\nabla} \cdot\left(\frac{1}{\rho_{r}(\boldsymbol{x})} \nabla p_{r}(\boldsymbol{x})\right)+\frac{\omega^{2}}{K_{r}(\boldsymbol{x})} p_{r}(\boldsymbol{x})=0
$$

In the equations above, $p_{r}$ is the phasor pressure field (in $\mathrm{N} / \mathrm{m}^{2}$ ). It is related to the time-harmonic pressure $\mathcal{P}_{r}$ by $\mathcal{P}_{r}(\boldsymbol{x}, t)=\operatorname{Re}\left\{p_{r}(\boldsymbol{x}) e^{j \omega t}\right\}$, where $\omega=2 \pi f$ is the angular frequency (in $\mathrm{rad} / \mathrm{s}$ ), $f$ is the frequency (in $\mathrm{Hz}$ ), and $\operatorname{Re}\{\cdot\}$ denotes the real part of a complex quantity. The density (in $\mathrm{kg} / \mathrm{m}^{3}$ ) and the bulk modulus (in Pa) within region $\Omega_{r}$ are given by the known functions $\rho_{r}: \Omega_{r} \rightarrow \mathbb{R}^{+}$and $K_{r}: \Omega_{r} \rightarrow \mathbb{R}^{+}$, respectively. We assume that the material properties of the host medium are constant, i.e., $\rho_{4}$ and $K_{4}$ are constant functions. These constants are used to normalize the density and bulk modulus for all other regions, i.e., we define 'relative' properties, and write, for $r=1, \ldots, 4$ and for $x \in \Omega_{r}$,

$$
\begin{aligned}
& \rho_{r, r e l}(x) \stackrel{\text { def }}{=} \rho_{r}(x) / \rho_{4}, \\
& K_{r, r e l}(x) \stackrel{\text { def }}{=} K_{r}(x) / K_{4} .
\end{aligned}
$$

The quantities $\rho_{r, r e l}$ and $K_{r, r e l}$ are dimensionless. It follows from these assumptions that $\rho_{4, r e l}=$ $K_{4, r e l}=1$ throughout the host medium $\Omega_{4}$. Substituting (4a) and (4b) in (3), we obtain new equations for the pressure fields: For each $r=1, \ldots, 4$, find $p_{r}: \bar{\Omega}_{r} \rightarrow \mathbb{C}$ such that for any $x \in \Omega_{r}$,

$$
\boldsymbol{\nabla} \cdot\left(\frac{1}{\rho_{r, r e l}(\boldsymbol{x})} \nabla p_{r}(\boldsymbol{x})\right)+\frac{k^{2}}{K_{r, r e l}(\boldsymbol{x})} p_{r}(\boldsymbol{x})=0
$$


where the wavenumber associated with the host medium is given by $k=\omega / c$ (in $\mathrm{rad} / \mathrm{m}$ ), and the speed of sound in the host medium is $c=\sqrt{K_{4} / \rho_{4}}$. The boundary condition to be satisfied by $p_{4}$ along $\Gamma_{R}$ is

$$
\nabla p_{4}(\boldsymbol{x}) \cdot \mathbf{n}_{4, \infty}(\boldsymbol{x})+\left(j k+\frac{1}{2 R}\right) p_{4}(\boldsymbol{x})=F(\boldsymbol{x}),
$$

for all $x \in \Gamma_{R}$, where $\mathbf{n}_{4, \infty}$ is the outward-pointing unit normal vector at $x$ (see Fig. 1), and the function $F$ is given by

$$
F(\boldsymbol{x}) \stackrel{\text { def }}{=} \nabla p^{i n c}(\boldsymbol{x}) \cdot \mathbf{n}_{4, \infty}(\boldsymbol{x})+\left(j k+\frac{1}{2 R}\right) p^{i n c}(\boldsymbol{x}),
$$

for all $x \in \Gamma_{R}$, where $p^{i n c}$ is the incident field. Eqs. (6a) and (6b) are derived after application of the firstorder Bayliss-Turkel absorbing boundary conditions along the circle $\Gamma_{R}$ [31]. Considering the interface conditions, we have the closed curves $\Gamma_{1,2}, \Gamma_{2,4}$, and $\Gamma_{3,4}$ in Fig. 1 . Along each of these interfaces, we impose the traditional equations of equilibrium (equal pressures on both sides of the interface) and compatibility (equal normal velocities). When the velocities are replaced by pressure gradients, we obtain jumps (or discontinuities) in their normal components, since the densities are different on the two sides of the interface.

\section{WEAK FORMS}

\subsection{Function spaces}

The problem in strong form is defined pointwise by Eqs. (5), complemented by the boundary condition (6a) and by the interface conditions. When looking for weak solutions, the fields are no longer defined pointwise, and must be sought within suitable Lebesgue and Sobolev spaces [32,33]. We therefore shall no longer consider the dependence of the fields on position $x$. The behavior of the pressure fields at the boundaries and interfaces is characterized by their traces, and we now assume that $p_{r}: \Omega_{r} \rightarrow \mathbb{C}$, for $r=1, \ldots, 4$. We look for weak solutions regular enough to satisfy $p_{r} \in H^{1}\left(\Omega_{r}\right)$, for $r=1, \ldots, 4$. Moreover, we assume material properties such that $\left(1 / \rho_{r, \text { rel }}\right) \in C\left(\bar{\Omega}_{r}\right)$ and $\left(1 / K_{r, \text { rel }}\right) \in C\left(\bar{\Omega}_{r}\right)$. For bounded domains $\Omega_{r}$ we have $C\left(\bar{\Omega}_{r}\right) \subset L^{\infty}\left(\Omega_{r}\right)$ (see, e.g., chapter 6 in [34]). For further details on the regularity of weak solutions to the Helmholtz equation, we refer to [35-37]. We need the following result, discussed in [13,38-40].

Theorem 3.1. Let $\Omega$ be a domain in $\mathbb{R}^{2}$ with Lipschitz continuous boundary $\partial \Omega$. Suppose that $u \in H^{1}(\Omega), \overline{\bar{\sigma}} \in$ $L^{\infty}(\Omega)^{2 \times 2}$, and $\overline{\bar{\sigma}} \cdot \nabla u \in H(\mathbf{d i v} ; \Omega)$. It can be concluded that

1. $\gamma_{\mathbf{n}, \partial \Omega}(\overline{\bar{\sigma}} \cdot \nabla u) \in H^{-1 / 2}(\partial \Omega)$.

2. For any $v \in H^{1}(\Omega), \int_{\Omega} v \nabla \cdot(\overline{\bar{\sigma}} \cdot \nabla u) d \Omega+\int_{\Omega} \nabla v \cdot(\overline{\bar{\sigma}} \cdot \nabla u) d \Omega=\left\langle\gamma_{\mathbf{n}, \partial \Omega}(\overline{\bar{\sigma}} \cdot \nabla u) \mid \gamma_{\partial \Omega}(v)\right\rangle_{H^{1 / 2}(\partial \Omega)}$,

where $\gamma_{\partial \Omega}(v) \in H^{1 / 2}(\partial \Omega)$ is the (interior) trace of $v$ along the boundary $\partial \Omega$, and $\gamma_{\mathbf{n}, \partial \Omega}(\overline{\bar{\sigma}} \cdot \nabla u)$ is the normal trace of $\overline{\bar{\sigma}} \cdot \nabla u$ along $\partial \Omega$. The brackets represent the duality pairing between the functional $\gamma_{\mathbf{n}, \partial \Omega}(\overline{\bar{\sigma}} \cdot \nabla u) \in$ $H^{-1 / 2}(\partial \Omega)$ and the function $\gamma_{\partial \Omega}(v) \in H^{1 / 2}(\partial \Omega)$.

In order to use this theorem, for $r=1, \ldots, 4$, we make the substitutions $\Omega=\Omega_{r}, u=p_{r}$, and $\overline{\bar{\sigma}}=\left(1 / \rho_{r, r e l}\right) \overline{\bar{I}}$, where $\overline{\bar{I}}$ is the identity tensor. Using the assumptions we made regarding the regularity of $1 / \rho_{r, \text { rel }}$ and $1 / K_{r, \text { rel }}$, it can be shown that $\overline{\bar{\sigma}} \in L^{\infty}\left(\Omega_{r}\right)^{2 \times 2}$ and that $\left(1 / \rho_{r, r e l}\right) \nabla p_{r} \in H\left(\operatorname{div} ; \Omega_{r}\right)$. We conclude from (7a) that the normal trace $\gamma_{\mathbf{n}, \partial \Omega_{r}}\left(\left(1 / \rho_{r, r e l}\right) \nabla p_{r}\right)$ belongs to $H^{-1 / 2}\left(\partial \Omega_{r}\right)$. The equations in weak form are obtained from (5) and (7b): For $r=1, \ldots, 4$ and for any $v_{r} \in H^{1}\left(\Omega_{r}\right)$,

$$
\int_{\Omega_{r}}\left(\frac{1}{\rho_{r, r e l}} \nabla v_{r} \cdot \nabla p_{r}-\frac{k^{2}}{K_{r, r e l}} v_{r} p_{r}\right) d \Omega-\left\langle\gamma_{\mathbf{n}, \partial \Omega_{r}}\left(\frac{1}{\rho_{r, r e l}} \nabla p_{r}\right) \mid \gamma \partial \Omega_{r}\left(v_{r}\right)\right\rangle_{H^{1 / 2}\left(\partial \Omega_{r}\right)}=0 .
$$


The following result is fundamental to justifying the form assumed by the boundary and interface conditions in the weak sense, when the region $\Omega_{r}$ is not simply-connected (as $\Omega_{2}$ and $\Omega_{4}$ in Fig. 1). We give the proof in Appendix C.

Theorem 3.2. Let $\Omega$ be an open, bounded, and not simply-connected region of $\mathbb{R}^{2}$ with Lipschitz continuous boundary $\partial \Omega$ (see example in Fig. 2). Suppose the boundary $\partial \Omega$ can be represented by the union of a number of connected components ("pieces") as $\partial \Omega=\sigma_{1} \cup \cdots \cup \sigma_{c}$, where $c>1$. These connected components are closed curves, and lie at a certain distance from each other. Consider the $k$-th connected component $\sigma_{k}$ of $\partial \Omega$, where $1 \leq k \leq c$. Let the extension by zero operator $E_{\left[\sigma_{k}, \partial \Omega\right]}$ be defined as: For any $w \in H^{1 / 2}\left(\sigma_{k}\right), E_{\left[\sigma_{k}, \partial \Omega\right]}(w)$ is a function defined on the whole boundary $\partial \Omega$ such that

$$
E_{\left[\sigma_{k}, \partial \Omega\right]}(w) \stackrel{\text { def }}{=}\left\{\begin{array}{lll}
w & \text { on } & \sigma_{i}, i=k \\
0 & \text { on } & \sigma_{i}, i \neq k
\end{array}\right.
$$

It can be concluded that:

1. $E_{\left[\sigma_{k}, \partial \Omega\right]}$ is a linear and bounded operator from $H^{1 / 2}\left(\sigma_{k}\right)$ into $H^{1 / 2}(\partial \Omega)$.

2. There is an operator $\Lambda_{\left[\partial \Omega, \sigma_{k}\right]}: H^{-1 / 2}(\partial \Omega) \rightarrow H^{-1 / 2}\left(\sigma_{k}\right)$ such that for any $\mu \in H^{-1 / 2}(\partial \Omega)$ and for any $w \in H^{1 / 2}\left(\sigma_{k}\right)$,

$$
\left\langle\Lambda_{\left[\partial \Omega, \sigma_{k}\right]}(\mu) \mid w\right\rangle_{H^{1 / 2}\left(\sigma_{k}\right)}=\left\langle\mu \mid E_{\left[\sigma_{k}, \partial \Omega\right]}(w)\right\rangle_{H^{1 / 2}(\partial \Omega)} .
$$

3. For any $\mu \in H^{-1 / 2}(\partial \Omega)$ and for any $g \in H^{1 / 2}(\partial \Omega)$,

$$
\langle\mu \mid g\rangle_{H^{1 / 2}(\partial \Omega)}=\sum_{k=1}^{c}\left\langle\Lambda_{\left[\partial \Omega, \sigma_{k}\right]}(\mu)|g|_{\sigma_{k}}\right\rangle_{H^{1 / 2}\left(\sigma_{k}\right)},
$$

where $\left.g\right|_{\sigma_{k}}$ is the restriction of $g$ to $\sigma_{k}$. Fig. 2 shows the geometrical notions involved in this theorem.

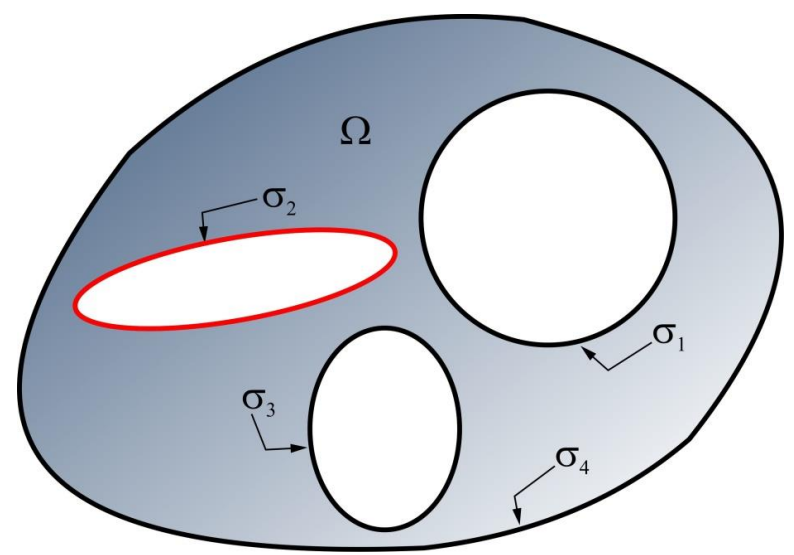

Fig. 2. Geometrical notions involved in Theorem 3.2. In this example, the region $\Omega$ is not simply-connected. Its boundary $\partial \Omega$ is composed of 4 connected components $\sigma_{1}, \ldots, \sigma_{4}$. These components are all closed curves, and do not touch each other. In this example, $c=4$, and we have $\partial \Omega=\sigma_{1} \cup \sigma_{2} \cup \sigma_{3} \cup \sigma_{4}$. We consider the second component $\sigma_{2}$, shown in red. Let $w$ be an arbitrary function whose domain of definition is $\sigma_{2}$. Suppose $w$ belongs to the space $H^{1 / 2}\left(\sigma_{2}\right)$. The new function $E_{\left[\sigma_{2}, \partial \Omega\right]}(w)$ in $(9 \mathrm{a})$ is defined in all of $\partial \Omega$; it coincides with $w$ at the points located along $\sigma_{2}$, and it is zero at the points located along $\sigma_{1}, \sigma_{3}$, and $\sigma_{4}$ (shown in black). Let $\mu$ be a functional to be paired with functions defined on the whole of $\partial \Omega$. Suppose $\mu$ belongs to $H^{-1 / 2}(\partial \Omega)$. The new functional $\Lambda_{\left[\partial \Omega, \sigma_{2}\right]}(\mu)$ in $(9 \mathrm{~b})$ belongs to $H^{-1 / 2}\left(\sigma_{2}\right)$, and must be paired with functions defined on $\sigma_{2}$ only

\subsection{Boundary conditions in the weak sense}

The objective of this subsection is to derive a variational expression to replace the pointwise condition (6a). 


\subsubsection{The incident field}

The function defined in (6b) in terms of the incident pressure field $p^{i n c}$ must be regular enough to belong to $L^{2}\left(\Gamma_{R}\right)$. It can be shown that incident fields given by plane wave solutions of the form $p^{i n c}(x)=e^{-j \boldsymbol{k} \cdot \boldsymbol{x}}$, when substituted in (6b), allow us to conclude that $\|F\|_{L^{2}\left(\Gamma_{R}\right)}<\infty$. In the expression of the plane wave, $\boldsymbol{k}=k \hat{k}$, where the unit vector $\hat{k}$ gives the direction of propagation of the plane wave, and $x=[x, y]$ is the radius vector.

\subsubsection{Variational expression}

Since $p_{4} \in H^{1}\left(\Omega_{4}\right)$ (see Section 3.1), its trace $\gamma \partial \Omega_{4}\left(p_{4}\right)$ belongs to $H^{1 / 2}\left(\partial \Omega_{4}\right)$ (see Theorem A.1 in Appendix A). Moreover, since $\Gamma_{R}$ is a subset of $\partial \Omega_{4}$ (see (2d)), it follows that the restriction of the trace to $\Gamma_{R}$ belongs to $H^{1 / 2}\left(\Gamma_{R}\right)$ (property 2 in Appendix B). Consider the bilinear form $g: H^{1 / 2}\left(\Gamma_{R}\right) \times$ $H^{1 / 2}\left(\Gamma_{R}\right) \rightarrow \mathbb{C}$ defined as: For any $(t, w) \in H^{1 / 2}\left(\Gamma_{R}\right) \times H^{1 / 2}\left(\Gamma_{R}\right)$,

$$
g(t, w) \stackrel{\text { def }}{=} \int_{\Gamma_{R}}\left(j k+\frac{1}{2 R}\right) t w d \Gamma .
$$

It can be shown that $g$ is bounded, i.e., we can find a positive constant $C$ such that $|g(t, w)| \leq$ $C\|t\|_{H^{1 / 2}\left(\Gamma_{R}\right)}\|w\|_{H^{1 / 2}\left(\Gamma_{R}\right)}$. If we fix the first coordinate, i.e., if we make $t=\left.\gamma_{\partial \Omega_{4}}\left(p_{4}\right)\right|_{\Gamma_{R}}$, then we can construct a linear and bounded functional

$$
G^{\prime}(w) \stackrel{\text { def }}{=} g\left(\left.\gamma_{\partial \Omega_{4}}\left(p_{4}\right)\right|_{\Gamma_{R}}, w\right)
$$

for any $w \in H^{1 / 2}\left(\Gamma_{R}\right)$, i.e., $G^{\prime} \in H^{-1 / 2}\left(\Gamma_{R}\right)$.

It can be shown that $G^{\prime}$ is bounded, i.e., we can find a positive constant $C$ (independent of $w$ ) such that $\left|G^{\prime}(w)\right| \leq C\|w\|_{H^{1 / 2}\left(\Gamma_{R}\right)}$. Since $G^{\prime}$ is linear and bounded, it follows that $G^{\prime} \in H^{-1 / 2}\left(\Gamma_{R}\right)$.

We introduce now another linear functional $F^{\prime}: H^{1 / 2}\left(\Gamma_{R}\right) \rightarrow \mathbb{C}$ defined as: For any $w \in H^{1 / 2}\left(\Gamma_{R}\right)$,

$$
F^{\prime}(w) \stackrel{\text { def }}{=} \int_{\Gamma_{R}} F w d \Gamma
$$

where the function $F$ is defined in (6b). If the incident field $p^{i n c}$ is assumed to be a plane wave, and using the fact that $\|F\|_{L^{2}\left(\Gamma_{R}\right)}<\infty$ (see Section 3.2.1), it is straightforward to show that $F^{\prime}$ is bounded. It thus follows that $F^{\prime} \in H^{-1 / 2}\left(\Gamma_{R}\right)$.

The region $\Omega_{4}$ corresponding to the host medium is not simply-connected (see Section 2.1). Moreover, we note that the normal trace $\gamma_{\mathbf{n}, \partial \Omega_{4}}\left(\left(1 / \rho_{4, \text { rel }}\right) \nabla p_{4}\right)$ belongs to $H^{-1 / 2}\left(\partial \Omega_{4}\right)$, see Section 3.1. Since $\rho_{4, \text { rel }}=1$ (see (4a)), we use (2d) and make the substitutions $\Omega=\Omega_{4}, \sigma_{k}=\Gamma_{R}$, and $\mu=\gamma_{\mathbf{n}, \partial \Omega_{4}}\left(\nabla p_{4}\right)$ in Theorem 3.2. The second conclusion (9b) allows us to conclude that there is an operator $\Lambda_{\left[\partial \Omega_{4}, \Gamma_{R}\right]}$ : $H^{-1 / 2}\left(\partial \Omega_{4}\right) \rightarrow H^{-1 / 2}\left(\Gamma_{R}\right)$ such that for any $w \in H^{1 / 2}\left(\Gamma_{R}\right)$,

$$
\left\langle\Lambda_{\left[\partial \Omega_{4}, \Gamma_{R}\right]}\left(\gamma_{\mathbf{n}, \partial \Omega_{4}}\left(\nabla p_{4}\right)\right) \mid w\right\rangle_{H^{1 / 2}\left(\Gamma_{R}\right)}=\left\langle\gamma_{\mathbf{n}, \partial \Omega_{4}}\left(\nabla p_{4}\right) \mid E_{\left[\Gamma_{R}, \partial \Omega_{4}\right]}(w)\right\rangle_{H^{1 / 2}\left(\partial \Omega_{4}\right)},
$$

where $E_{\left[\Gamma_{R}, \partial \Omega_{4}\right]}$ takes a function $w$ defined on $\Gamma_{R}$ and extends it by zero to the rest of the boundary $\partial \Omega_{4}$ (i.e., along the connected components $\Gamma_{2,4}$ and $\Gamma_{3,4}$, see (2d)). The boundary condition in pointwise sense (6a) is substituted by the operator equation:

$$
\Lambda_{\left[\partial \Omega_{4}, \Gamma_{R}\right]}\left(\gamma_{\mathbf{n}, \partial \Omega_{4}}\left(\nabla p_{4}\right)\right)+G^{\prime}=F^{\prime},
$$

in $H^{-1 / 2}\left(\Gamma_{R}\right)$, where $G^{\prime}$ and $F^{\prime}$ are defined in (10b) and (11), respectively. Eq. (13) immediately gives our desired variational expression once we form the duality pairings with elements from the space $H^{1 / 2}\left(\Gamma_{R}\right)$.

\subsection{Interface conditions in the weak sense}

We must now derive suitable variational expressions for the interface conditions that will replace those defined in a pointwise sense (see [10]). 


\subsubsection{The first set of interface conditions}

There are three interfaces in the geometry of Fig. 1, namely, the closed curves $\Gamma_{1,2}, \Gamma_{2,4}$, and $\Gamma_{3,4}$. Continuity of the pressure fields across these interfaces can be imposed in the weak sense through the expressions

$$
\begin{gathered}
\left\langle\mu\left|\gamma \partial \Omega_{2}\left(p_{2}\right)\right|_{\Gamma_{1,2}}-\gamma \partial \Omega_{1}\left(p_{1}\right)\right\rangle_{H^{1 / 2}\left(\Gamma_{1,2}\right)}=0, \text { for any } \mu \in H^{-1 / 2}\left(\Gamma_{1,2}\right), \\
\left\langle\mu\left|\gamma_{\partial \Omega_{4}}\left(p_{4}\right)\right|_{\Gamma_{2,4}}-\left.\gamma_{\partial \Omega_{2}}\left(p_{2}\right)\right|_{\Gamma_{2,4}}\right\rangle_{H^{1 / 2}\left(\Gamma_{2,4}\right)}=0, \text { for any } \mu \in H^{-1 / 2}\left(\Gamma_{2,4}\right), \\
\left\langle\mu\left|\gamma \partial \Omega_{4}\left(p_{4}\right)\right|_{\Gamma_{3,4}}-\gamma_{\partial \Omega_{3}}\left(p_{3}\right)\right\rangle_{H^{1 / 2}\left(\Gamma_{3,4}\right)}=0, \text { for any } \mu \in H^{-1 / 2}\left(\Gamma_{3,4}\right),
\end{gathered}
$$

where, for the case of not simply connected regions, $\left.\gamma \partial \Omega_{2}\left(p_{2}\right)\right|_{\Gamma_{1,2}}$ means the trace of $p_{2}$ (originally defined along the whole of boundary $\partial \Omega_{2}$ ) restricted to $\Gamma_{1,2}$, and similarly for the others.

\subsubsection{The second set of interface conditions}

We consider first the interface $\Gamma_{1,2}$, between regions $\Omega_{1}$ and $\Omega_{2}$. According to Section 3.1, the normal trace $\gamma_{\mathbf{n}, \partial \Omega_{1}}\left(\left(1 / \rho_{1, \text { rel }}\right) \nabla p_{1}\right)$ belongs to $H^{-1 / 2}\left(\partial \Omega_{1}\right)$, or to $H^{-1 / 2}\left(\Gamma_{1,2}\right)$, since $\partial \Omega_{1}=\Gamma_{1,2}$, by (2a). Still in accordance with Section 3.1, the normal trace $\gamma_{\mathbf{n}, \partial \Omega_{2}}\left(\left(1 / \rho_{2, \text { rel }}\right) \nabla p_{2}\right)$ belongs to $H^{-1 / 2}\left(\partial \Omega_{2}\right)$. In order to "restrict" this functional to $\Gamma_{1,2}$, we apply Theorem 3.2. We use (2b) and make the substitutions $\Omega=\Omega_{2}, \sigma_{k}=\Gamma_{1,2}$, and $\mu=\gamma_{\mathbf{n}, \partial \Omega_{2}}\left(\left(1 / \rho_{2, \text { rel }}\right) \nabla p_{2}\right)$ in Theorem 3.2. From the second conclusion (9b), we conclude that there is an operator $\Lambda_{\left[\partial \Omega_{2}, \Gamma_{1,2}\right]}: H^{-1 / 2}\left(\partial \Omega_{2}\right) \rightarrow H^{-1 / 2}\left(\Gamma_{1,2}\right)$ such that for any $w \in H^{1 / 2}\left(\Gamma_{1,2}\right)$,

$$
\left\langle\Lambda_{\left[\partial \Omega_{2}, \Gamma_{1,2}\right]}\left(\gamma_{\mathbf{n}, \partial \Omega_{2}}\left(\frac{1}{\rho_{2, r e l}} \nabla p_{2}\right)\right) \mid w\right\rangle_{H^{1 / 2}\left(\Gamma_{1,2}\right)}=\left\langle\gamma_{\mathbf{n}, \partial \Omega_{2}}\left(\frac{1}{\rho_{2, \text { rel }}} \nabla p_{2}\right) \mid E_{\left[\Gamma_{1,2}, \partial \Omega_{2}\right]}(w)\right\rangle_{H^{1 / 2}\left(\partial \Omega_{2}\right)},
$$

where $E_{\left[\Gamma_{1,2}, \partial \Omega_{2}\right]}$ takes a function $w$ defined on $\Gamma_{1,2}$ and extends it by zero to the rest of the boundary $\partial \Omega_{2}$ (i.e., along the connected component $\Gamma_{2,4}$, see $(2 b)$ ). The second interface condition along $\Gamma_{1,2}$ is substituted by the operator equation

$$
\gamma_{\mathbf{n}, \partial \Omega_{1}}\left(\frac{1}{\rho_{1, r e l}} \nabla p_{1}\right)+\Lambda_{\left[\partial \Omega_{2}, \Gamma_{1,2}\right]}\left(\gamma_{\mathbf{n}, \partial \Omega_{2}}\left(\frac{1}{\rho_{2, r e l}} \nabla p_{2}\right)\right)=0, \quad \text { in } H^{-1 / 2}\left(\Gamma_{1,2}\right),
$$

which immediately gives our desired variational expression once we form the duality pairing with elements from the space $H^{1 / 2}\left(\Gamma_{1,2}\right)$. The second interface conditions along $\Gamma_{2,4}$ and $\Gamma_{3,4}$ are derived by a similar reasoning, and are given by the operator equations:

$$
\begin{gathered}
\Lambda_{\left[\partial \Omega_{2,}, \Gamma_{2,4}\right]}\left(\gamma_{\mathbf{n}, \partial \Omega_{2}}\left(\frac{1}{\rho_{2, \text { rel }}} \nabla p_{2}\right)\right)+\Lambda_{\left[\partial \Omega_{4}, \Gamma_{2,4}\right]}\left(\gamma_{\mathbf{n}, \partial \Omega_{4}}\left(\nabla p_{4}\right)\right)=0, \text { in } H^{-1 / 2}\left(\Gamma_{2,4}\right), \\
\gamma_{\mathbf{n}, \partial \Omega_{3}}\left(\frac{1}{\rho_{3, \text { rel }}} \nabla p_{3}\right)+\Lambda_{\left[\partial \Omega_{4}, \Gamma_{3,4}\right]}\left(\gamma_{\mathbf{n}, \partial \Omega_{4}}\left(\nabla p_{4}\right)\right)=0, \quad \text { in } H^{-1 / 2}\left(\Gamma_{3,4}\right),
\end{gathered}
$$

where we recalled that $\rho_{4, \text { rel }}=1$ (see (4a)). Eqs. (15b) and (15c) give variational expressions once we form the duality pairings with elements from the spaces $H^{1 / 2}\left(\Gamma_{2,4}\right)$ and $H^{1 / 2}\left(\Gamma_{3,4}\right)$, respectively.

\subsection{The problem in weak form}

In this section we show how the Lagrange multiplier fields arise naturally and we present the final formulation of the problem in mixed form.

\subsubsection{Function spaces}

Given the geometry from Fig. 1, the suitable function spaces to be used in the weak formulation of the problem are given by

$$
\begin{aligned}
& \mathcal{X} \stackrel{\text { def }}{=} H^{1}\left(\Omega_{1}\right) \times H^{1}\left(\Omega_{2}\right) \times H^{1}\left(\Omega_{3}\right) \times H^{1}\left(\Omega_{4}\right), \\
& \mathcal{Y} \stackrel{\text { def }}{=} H^{-1 / 2}\left(\Gamma_{1,2}\right) \times H^{-1 / 2}\left(\Gamma_{2,4}\right) \times H^{-1 / 2}\left(\Gamma_{3,4}\right) .
\end{aligned}
$$


3.4.2. Lagrange multiplier fields

The first step is to sum equations (8) for $r=1, \ldots, 4$, which we write as

$\sum_{r=1}^{4} \int_{\Omega_{r}}\left(\frac{1}{\rho_{r, r e l}} \nabla v_{r} \cdot \nabla p_{r}-\frac{k^{2}}{K_{r, r e l}} v_{r} p_{r}\right) d \Omega-\sum_{r=1}^{4}\left\langle\gamma_{\mathbf{n}, \partial \Omega_{r}}\left(\frac{1}{\rho_{r, r e l}} \nabla p_{r}\right) \mid \gamma_{\partial \Omega_{r}}\left(v_{r}\right)\right\rangle_{H^{1 / 2}\left(\partial \Omega_{r}\right)}=0$,

for any $\left(v_{1}, v_{2}, v_{3}, v_{4}\right) \in \mathcal{X}$. Regions $\Omega_{2}$ and $\Omega_{4}$ are not simply connected (see (2b) and (2d)), and we use this fact together with the third conclusion (9c) from Theorem 3.2 to rewrite the above equation as

$$
\begin{aligned}
& \sum_{r=1}^{4} \int_{\Omega_{r}}\left(\frac{1}{\rho_{r, r e l}} \nabla v_{r} \cdot \nabla p_{r}-\frac{k^{2}}{K_{r, r e l}} v_{r} p_{r}\right) d \Omega-\langle\underbrace{\gamma_{\mathbf{n}, \partial \Omega_{1}}\left(\frac{1}{\rho_{1, r e l}} \nabla p_{1}\right)}_{\lambda_{1,2}} \mid \gamma_{\partial \Omega_{1}}\left(v_{1}\right)\rangle \\
& H^{1 / 2} \underbrace{\left(\partial \Omega_{1}\right)}_{\Gamma_{1,2}} \\
& -\left\langle\Lambda_{\left[\partial \Omega_{2}, \Gamma_{1,2}\right]}\left(\gamma_{\mathbf{n}, \partial \Omega_{2}}\left(\frac{1}{\rho_{2, \text { rel }}} \nabla p_{2}\right)\right)\left|\gamma_{\partial \Omega_{2}}\left(v_{2}\right)\right|_{\Gamma_{1,2}}\right\rangle_{H^{1 / 2}\left(\Gamma_{1,2}\right)} \\
& -\langle\underbrace{\Lambda \Lambda_{\left[\Omega_{2}, \Gamma_{2,4}\right]}\left(\gamma_{\mathbf{n}, \partial \Omega_{2}}\left(\frac{1}{\rho_{2, r e l}} \nabla p_{2}\right)\right)}_{\lambda_{2,4}}\left|\gamma_{\partial \Omega_{2}}\left(v_{2}\right)\right|_{\Gamma_{2,4}}\rangle \\
& H^{1 / 2}\left(\Gamma_{2,4}\right) \\
& -\langle\underbrace{\gamma_{\mathbf{n}, \partial \Omega_{3}}\left(\frac{1}{\rho_{3, r e l}} \nabla p_{3}\right)}_{\lambda_{3,4}} \mid \gamma_{\partial \Omega_{3}}\left(v_{3}\right)\rangle \\
& H^{1 / 2} \underbrace{\left(\partial \Omega_{3}\right)}_{\Gamma_{3,4}} \\
& -\left\langle\Lambda_{\left[\partial \Omega_{4} \Gamma_{2,4}\right]}\left(\gamma_{\mathbf{n}, \partial \Omega_{4}}\left(\nabla p_{4}\right)\right)\left|\gamma_{\partial \Omega_{4}}\left(v_{4}\right)\right|_{\Gamma_{2,4}}\right\rangle_{H^{1 / 2}\left(\Gamma_{2,4}\right)} \\
& -\left\langle\Lambda_{\left[\partial \Omega_{4} \Gamma_{3,4}\right]}\left(\gamma_{\mathbf{n}, \partial \Omega_{4}}\left(\nabla p_{4}\right)\right)\left|\gamma_{\partial \Omega_{4}}\left(v_{4}\right)\right|_{\Gamma_{3,4}}\right\rangle_{H^{1 / 2}\left(\Gamma_{3,4}\right)} \\
& -\left\langle\Lambda_{\left[\partial \Omega_{4} \Gamma_{R}\right]}\left(\gamma_{\mathbf{n}, \partial \Omega_{4}}\left(\nabla p_{4}\right)\right)\left|\gamma_{\partial \Omega_{4}}\left(v_{4}\right)\right|_{\Gamma_{R}}\right\rangle_{H^{1 / 2}\left(\Gamma_{R}\right)}=0,
\end{aligned}
$$

for any $\left(v_{1}, v_{2}, v_{3}, v_{4}\right) \in \mathcal{X}$, where we recalled that $\rho_{4, \text { rel }}=1$, see (4a). Secondly, in (17a) we make the substitutions $\partial \Omega_{1}=\Gamma_{1,2}$ and $\partial \Omega_{3}=\Gamma_{3,4}$, according to (2a) and (2c), respectively. These are indicated above. Thirdly, we introduce the Lagrange multiplier fields

$$
\begin{aligned}
& \lambda_{1,2} \stackrel{\text { def }}{=} \gamma_{\mathbf{n}, \partial \Omega_{1}}\left(\frac{1}{\rho_{1, \text { rel }}} \nabla p_{1}\right), \\
& \lambda_{2,4} \stackrel{\text { def }}{=} \Lambda_{\left[\partial \Omega_{2}, \Gamma_{2,4}\right]}\left(\gamma_{\mathbf{n}, \partial \Omega_{2}}\left(\frac{1}{\rho_{2, \text { rel }}} \nabla p_{2}\right)\right), \\
& \lambda_{3,4} \stackrel{\text { def }}{=} \gamma_{\mathbf{n}, \partial \Omega_{3}}\left(\frac{1}{\rho_{3, \text { rel }}} \nabla p_{3}\right),
\end{aligned}
$$

which are also indicated in (17a). Note that these Lagrange multiplier fields are defined differently from the definitions in other problem formulations, see e.g. [6,14]. The fourth step is to combine (15a) with $(17 b),(15 b)$ with (17c), and (15c) with (17d), and substitute back in (17a). The result becomes

$$
\begin{aligned}
& \sum_{r=1}^{4} \int_{\Omega_{r}}\left(\frac{1}{\rho_{r, r e l}} \nabla v_{r} \cdot \nabla p_{r}-\frac{k^{2}}{K_{r, r e l}} v_{r} p_{r}\right) d \Omega+\left\langle\lambda_{1,2}\left|\gamma \partial \Omega_{2}\left(v_{2}\right)\right|_{\Gamma_{1,2}}-\gamma \partial \Omega_{1}\left(v_{1}\right)\right\rangle_{H^{1 / 2}\left(\Gamma_{1,2}\right)} \\
& +\left\langle\lambda_{2,4}\left|\gamma \partial \Omega_{4}\left(v_{4}\right)\right|_{\Gamma_{2,4}}-\left.\gamma \partial \Omega_{2}\left(v_{2}\right)\right|_{\Gamma_{2,4}}\right\rangle_{H^{1 / 2}\left(\Gamma_{2,4}\right)}+\left\langle\lambda_{3,4}\left|\gamma \partial \Omega_{4}\left(v_{4}\right)\right|_{\Gamma_{3,4}}-\gamma \partial \Omega_{3}\left(v_{3}\right)\right\rangle_{H^{1 / 2}\left(\Gamma_{3,4}\right)} \\
& -\left\langle\Lambda_{\left[\partial \Omega_{4}, \Gamma_{R}\right]}\left(\gamma_{n, \partial \Omega_{4}}\left(\nabla p_{4}\right)\right)\left|\gamma \partial \Omega_{4}\left(v_{4}\right)\right|_{\Gamma_{R}}\right\rangle_{H^{1 / 2}\left(\Gamma_{R}\right)}=0,
\end{aligned}
$$


for any $\left(v_{1}, v_{2}, v_{3}, v_{4}\right) \in \mathcal{X}$. The fifth and final step is to substitute the boundary condition (13) into (18).

\subsubsection{Mixed formulation}

We use bold letters to represent the vectors in spaces $\mathcal{X}$ and $\mathcal{Y}$, like $\mathbf{v}=\left(v_{1}, v_{2}, v_{3}, v_{4}\right)$ and $\mu=$ $\left(\mu_{1,2}, \mu_{2,4}, \mu_{3,4}\right)$, respectively. Defining the bilinear forms $a: \mathcal{X} \times \mathcal{X} \longrightarrow \mathbb{C}, b: \mathcal{X} \times \mathcal{Y} \longrightarrow \mathbb{C}$ and the linear functional $Q^{\prime}: \mathcal{X} \longrightarrow \mathbb{C}$ given by

$$
\begin{aligned}
& a(\mathbf{w}, \mathbf{v}) \stackrel{\text { def }}{=} \sum_{r=1}^{4} \int_{\Omega_{r}}\left(\frac{1}{\rho_{r, r e l}} \nabla v_{r} \cdot \nabla w_{r}-\frac{k^{2}}{K_{r, r e l}} v_{r} w_{r}\right) d \Omega \\
&+g\left(\left.\gamma \partial \Omega_{4}\left(w_{4}\right)\right|_{\Gamma_{R}},\left.\gamma \partial \Omega_{4}\left(v_{4}\right)\right|_{\Gamma_{R}}\right), \text { for any }(\mathbf{w}, \mathbf{v}) \in \mathcal{X} \times \mathcal{X}, \\
& b(\mathbf{v}, \boldsymbol{\mu}) \stackrel{\text { def }}{=}\langle\left.\mu_{1,2}\left|\gamma \partial \Omega_{2}\left(v_{2}\right)\right|_{\Gamma_{1,2}}-\gamma \partial \Omega_{1}\left(v_{1}\right)\right\rangle_{H^{1 / 2}\left(\Gamma_{1,2}\right)} \\
&+\left\langle\mu_{2,4}\left|\gamma \partial \Omega_{4}\left(v_{4}\right)\right|_{\Gamma_{2,4}}-\left.\gamma_{\partial \Omega_{2}}\left(v_{2}\right)\right|_{\Gamma_{2,4}}\right\rangle_{H^{1 / 2}\left(\Gamma_{2,4}\right)} \\
&+\left\langle\mu_{3,4}\left|\gamma \partial \Omega_{4}\left(v_{4}\right)\right|_{\Gamma_{3,4}}-\gamma \partial \Omega_{3}\left(v_{3}\right)\right\rangle_{H^{1 / 2}\left(\Gamma_{3,4}\right)^{\prime}} \text { for any }(\mathbf{v}, \boldsymbol{\mu}) \in \mathcal{X} \times \mathcal{Y}, \\
& Q^{\prime}(\mathbf{v}) \stackrel{\text { def }}{=}\left\langle F^{\prime}\left|\gamma \partial \Omega_{4}\left(v_{4}\right)\right|_{\Gamma_{R}}\right\rangle_{H^{1 / 2}\left(\Gamma_{R}\right)^{\prime}} \text { for any } \mathbf{v} \in \mathcal{X},
\end{aligned}
$$

Eq. (18) becomes

$$
a(\mathbf{p}, \mathbf{v})+b(\mathbf{v}, \lambda)=\left\langle Q^{\prime} \mid \mathbf{v}\right\rangle_{\mathcal{X}^{\prime}}
$$

for any $\mathbf{v} \in \mathcal{X}$. It can be shown that the functional $Q^{\prime}$ defined in (19c) is bounded in the norm of $\mathcal{X}$ (see (29a)). The field equations (8), the boundary condition (13), and the second set of interface conditions (15a)-(15c) are all embedded in (20a). Finally we impose the first set of interface conditions given by (14a)-(14c). If we consider arbitrary functionals $\mu_{1,2} \in H^{-1 / 2}\left(\Gamma_{1,2}\right), \mu_{2,4} \in H^{-1 / 2}\left(\Gamma_{2,4}\right)$, and $\mu_{3,4} \in H^{-1 / 2}\left(\Gamma_{3,4}\right)$, substitute them for $\mu$ in (14a), (14b), and (14c), respectively, and sum the result, we obtain

$$
b(\mathbf{p}, \boldsymbol{\mu})=0, \quad \text { for any } \boldsymbol{\mu} \in \mathcal{Y} .
$$

When Eqs. (20a) and (20b) are put together, our problem can formally be written as

$$
\begin{aligned}
& \text { Find }(\mathbf{p}, \boldsymbol{\lambda}) \in \mathcal{X} \times \mathcal{Y} \text { such that } \\
& a(\mathbf{p}, \boldsymbol{v})+b(\mathbf{v}, \boldsymbol{\lambda})=\left\langle Q^{\prime} \mid \mathbf{v}\right\rangle_{\mathcal{X}}, \quad \text { for any } \mathbf{v} \in \mathcal{X}, \\
& b(\mathbf{p}, \boldsymbol{\mu})=0, \quad \text { for any } \boldsymbol{\mu} \in \mathcal{Y},
\end{aligned}
$$

whose structure is readily recognized to be that of a mixed (saddle-point) formulation $[6,12,23]$.

\section{DISCRETIZATION IN THE METHOD OF FINITE SPHERES}

Given an arbitrary region $\Omega$ (like one of the regions $\Omega_{1}, \ldots, \Omega_{4}$ in Fig. 1), we proceed to cover it with balls, or spheres, as illustrated in Fig. $3[1,10]$. Next, a collection of real-valued basis functions is constructed; each basis function has the whole set $\Omega$ as its domain of definition, but it is different from zero only within a certain ball. These compactly-supported basis functions lead to sparse matrices, as in traditional finite element analysis, whose basis functions are nonzero only within certain elements in the mesh [6]. These basis functions allow us to construct finite-dimensional subspaces $\mathcal{M}\left(\Omega_{r}\right) \subset H^{1}\left(\Omega_{r}\right)$ for $r=1, \ldots, 4$. Arbitrary functions $v_{r} \in H^{1}\left(\Omega_{r}\right)$ can be approximated by their discretized equivalents $v_{r ; h} \in \mathcal{M}\left(\Omega_{r}\right)$ as

$$
\begin{aligned}
& v_{r ; h}=\mathbf{H}_{r}^{T} \tilde{\mathbf{V}}_{r}, \\
& \gamma_{\partial \Omega_{r}}\left(v_{r ; h}\right)=\mathbf{T}_{r}^{T} \tilde{\mathbf{V}}_{r},
\end{aligned}
$$


where $\mathbf{H}_{r}: \Omega_{r} \longrightarrow \mathbb{R}^{\operatorname{dim} \mathcal{M}\left(\Omega_{r}\right)}$ is a vector function collecting all basis functions defined on $\Omega_{r}$, and $\mathbf{T}_{r}: \partial \Omega_{r} \longrightarrow \mathbb{R}^{\operatorname{dim} \mathcal{M}\left(\Omega_{r}\right)}$ is a vector function collecting the traces of all basis functions along the boundary $\partial \Omega_{r}$ [10]. The vector $\tilde{\mathbf{V}}_{r} \in \mathbb{C}^{\operatorname{dim} \mathcal{M}\left(\Omega_{r}\right)}$ collects the expansion coefficients. After the finite- dimensional subspaces $\mathcal{M}\left(\Omega_{r}\right)$ have been constructed for $r=1, \ldots, 4$, we use $\mathcal{X}_{h} \stackrel{\text { def }}{=} \mathcal{M}\left(\Omega_{1}\right) \times \mathcal{M}\left(\Omega_{2}\right) \times$ $\mathcal{M}\left(\Omega_{3}\right) \times \mathcal{M}\left(\Omega_{4}\right)$ as the finite-dimensional counterpart to space $\mathcal{X}$ introduced in (16a), where we have for the dimensions of these finite-dimensional subspaces, $\operatorname{dim} \mathcal{X}_{h}=\operatorname{dim} \mathcal{M}\left(\Omega_{1}\right)+\ldots+\operatorname{dim} \mathcal{M}\left(\Omega_{4}\right)$. Arbitrary elements of $\mathcal{X}_{h}$ can be represented by vectors as $\mathbf{v}_{h}=\left(v_{1 ; h}, v_{2 ; h}, v_{3 ; h}, v_{4 ; h}\right)$.

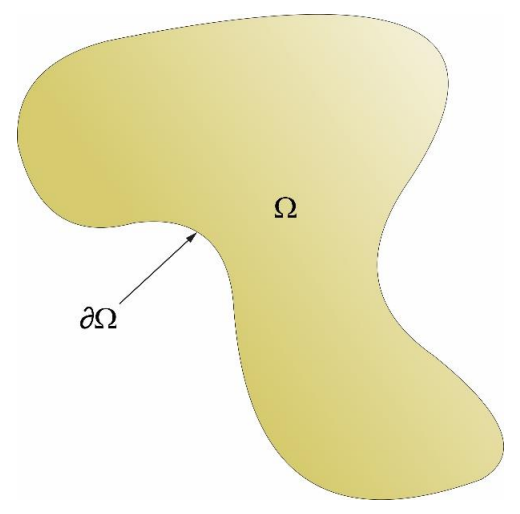

(a)

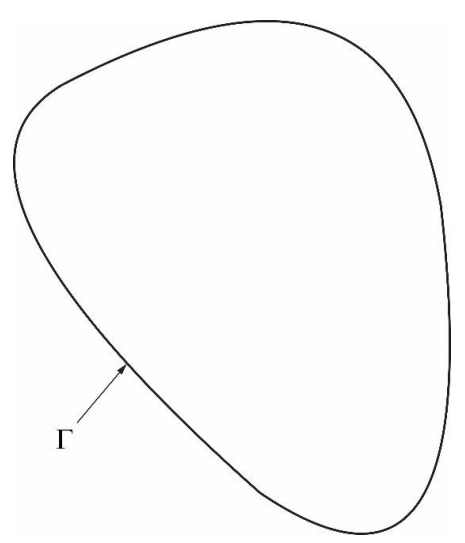

(c)

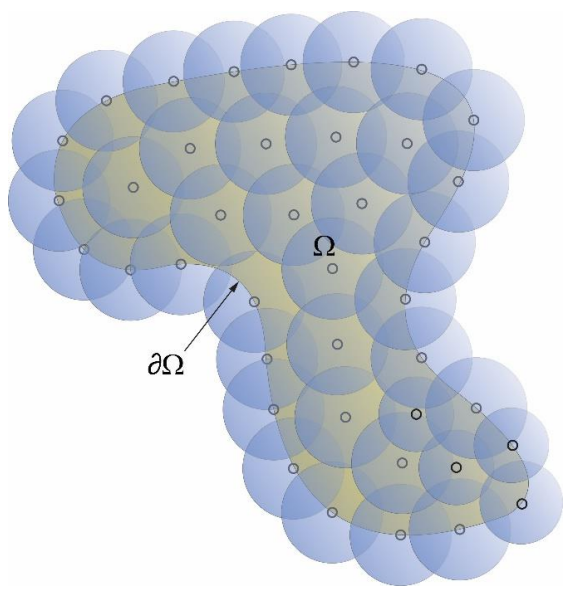

(b)

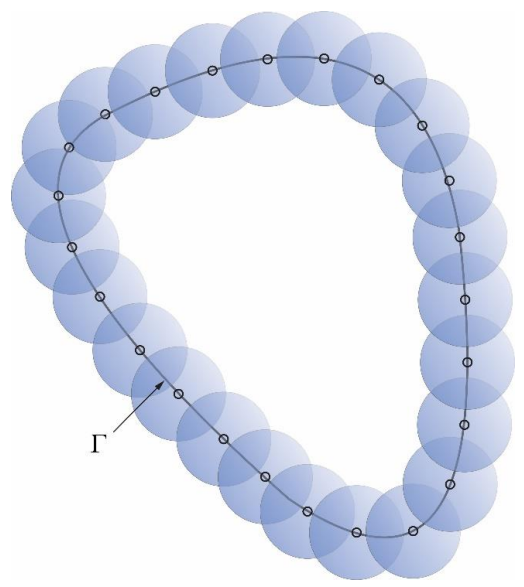

(d)

Fig. 3. (a) In the method of finite spheres, an arbitrary region $\Omega$ together with its boundary $\partial \Omega$ must be covered by a finite collection of balls, as in (b). The same holds for closed curves: (c) Given an arbitrary closed curve $\Gamma$, it must be covered by a finite collection of balls, as in $(\mathrm{d})$

The construction of discrete spaces for the Lagrange multiplier fields defined at the interfaces is similar. An arbitrary closed curve $\Gamma$ (like one of the curves $\Gamma_{1,2}, \Gamma_{2,4}$, and $\Gamma_{3,4}$ in Fig. 1) is also covered with balls, as in Fig. 3. Given arbitrary functionals $\mu_{1,2} \in H^{-1 / 2}\left(\Gamma_{1,2}\right), \mu_{2,4} \in H^{-1 / 2}\left(\Gamma_{2,4}\right)$, and $\mu_{3,4} \in H^{-1 / 2}\left(\Gamma_{3,4}\right)$, their finite-dimensional counterparts are represented by $\mu_{1,2 ; h} \in \mathcal{M}\left(\Gamma_{1,2}\right)^{\prime}, \mu_{2,4 ; h} \in$ $\mathcal{M}\left(\Gamma_{2,4}\right)^{\prime}$, and $\mu_{3,4 ; h} \in \mathcal{M}\left(\Gamma_{3,4}\right)^{\prime}$, respectively. The spaces $\mathcal{M}\left(\Gamma_{1,2}\right)^{\prime}, \mathcal{M}\left(\Gamma_{2,4}\right)^{\prime}$, and $\mathcal{M}\left(\Gamma_{3,4}\right)^{\prime}$ are finitedimensional subspaces of $H^{-1 / 2}\left(\Gamma_{1,2}\right), H^{-1 / 2}\left(\Gamma_{2,4}\right)$, and $H^{-1 / 2}\left(\Gamma_{3,4}\right)$, respectively. The action of the 
discretized functionals $\mu_{1,2 ; h}, \mu_{2,4 ; h}$, and $\mu_{3,4 ; h}$ on arbitrary functions is given by

$$
\begin{aligned}
& \left\langle\mu_{1,2 ; h} \mid w\right\rangle_{H^{1 / 2}\left(\Gamma_{1,2}\right)}=\tilde{\mathbf{U}}_{1,2}^{T} \int_{\Gamma_{1,2}} \mathbf{H}_{1,2} w d \Gamma, \quad \text { for any } w \in H^{1 / 2}\left(\Gamma_{1,2}\right), \\
& \left\langle\mu_{2,4 ; h} \mid w\right\rangle_{H^{1 / 2}\left(\Gamma_{2,4}\right)}=\tilde{\mathbf{U}}_{2,4}^{T} \int_{\Gamma_{2,4}} \mathbf{H}_{2,4} w d \Gamma, \quad \text { for any } w \in H^{1 / 2}\left(\Gamma_{2,4}\right), \\
& \left\langle\mu_{3,4 ; h} \mid w\right\rangle_{H^{1 / 2}\left(\Gamma_{3,4}\right)}=\tilde{\mathbf{U}}_{3,4}^{T} \int_{\Gamma_{3,4}} \mathbf{H}_{3,4} w d \Gamma, \quad \text { for any } w \in H^{1 / 2}\left(\Gamma_{3,4}\right),
\end{aligned}
$$

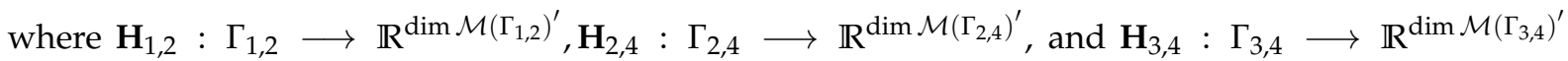
are vector functions which collect suitable basis functions defined along the curves $\Gamma_{1,2}, \Gamma_{2,4}$, and $\Gamma_{3,4}$,

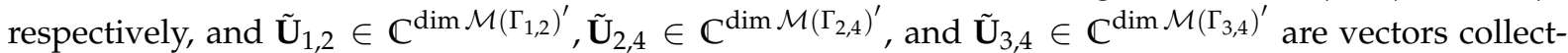
ing the expansion coefficients [10]. After the three finite-dimensional subspaces $\mathcal{M}\left(\Gamma_{1,2}\right)^{\prime}, \mathcal{M}\left(\Gamma_{2,4}\right)^{\prime}$, and $\mathcal{M}\left(\Gamma_{3,4}\right)^{\prime}$ have been constructed we use $\mathcal{Y}_{h} \stackrel{\text { def }}{=} \mathcal{M}\left(\Gamma_{1,2}\right)^{\prime} \times \mathcal{M}\left(\Gamma_{2,4}\right)^{\prime} \times \mathcal{M}\left(\Gamma_{3,4}\right)^{\prime}$ as the finitedimensional counterpart to space $\mathcal{Y}$ introduced in (16b). Its dimension is given by $\operatorname{dim} \mathcal{Y}_{h}=$ $\operatorname{dim} \mathcal{M}\left(\Gamma_{1,2}\right)^{\prime}+\operatorname{dim} \mathcal{M}\left(\Gamma_{2,4}\right)^{\prime}+\operatorname{dim} \mathcal{M}\left(\Gamma_{3,4}\right)^{\prime}$. Arbitrary elements of $\mathcal{Y}_{h}$ can be represented by vectors as $\mu_{h}=\left(\mu_{1,2 ; h}, \mu_{2,4 ; h}, \mu_{3,4 ; h}\right)$.

After all pressure fields $p_{1}, \ldots, p_{4}$, test functions $v_{1}, \ldots, v_{4}$, Lagrange multiplier fields $\lambda_{1,2}, \lambda_{2,4}, \lambda_{3,4}$, and test functionals $\mu_{1,2}, \mu_{2,4}, \mu_{3,4}$ have been substituted in (21) by their finite-dimensional counterparts defined by the expansions (22a)-(22c), we obtain the discrete mixed problem

$$
\begin{aligned}
& \text { Find }\left(\mathbf{p}_{h}, \lambda_{h}\right) \in \mathcal{X}_{h} \times \mathcal{Y}_{h} \text { such that } \\
& a\left(\mathbf{p}_{h}, \mathbf{v}_{h}\right)+b\left(\mathbf{v}_{h}, \lambda_{h}\right)=\left\langle Q^{\prime} \mid \mathbf{v}_{h}\right\rangle_{\mathcal{X}}, \quad \text { for any } \mathbf{v}_{h} \in \mathcal{X}_{h}, \\
& b\left(\mathbf{p}_{h}, \boldsymbol{\mu}_{h}\right)=0, \quad \text { for any } \boldsymbol{\mu}_{h} \in \mathcal{Y}_{h} .
\end{aligned}
$$

The individual terms in (23) assume the representation

$$
\begin{aligned}
& a\left(\mathbf{p}_{h}, \mathbf{v}_{h}\right)=\tilde{\mathbf{V}}^{T} \bar{A} \tilde{\mathbf{P}}, \\
& b\left(\mathbf{v}_{h}, \boldsymbol{\lambda}_{h}\right)=\tilde{\mathbf{L}}^{T} \overline{\boldsymbol{B}} \tilde{\mathbf{V}}, \\
& \left\langle Q^{\prime} \mid \mathbf{v}_{h}\right\rangle_{\mathcal{X}}=\tilde{\mathbf{V}}^{T} \boldsymbol{F}, \\
& b\left(\mathbf{p}_{h}, \boldsymbol{\mu}_{h}\right)=\tilde{\mathbf{U}}^{T} \overline{\boldsymbol{B}} \tilde{\mathbf{P}} .
\end{aligned}
$$

The vectors $\tilde{\mathbf{P}}^{T}=\left[\tilde{\mathbf{P}}_{1}^{T}, \tilde{\mathbf{P}}_{2}^{T}, \tilde{\mathbf{P}}_{3}^{T}, \tilde{\mathbf{P}}_{4}^{T}\right]$ and $\tilde{\mathbf{V}}^{T}=\left[\tilde{\mathbf{V}}_{1}^{T}, \tilde{\mathbf{V}}_{2}^{T}, \tilde{\mathbf{V}}_{3}^{T}, \tilde{\mathbf{V}}_{4}^{T}\right]$ collect all coefficients used in the expansion of $p_{1 ; h}, \ldots, p_{4 ; h}$ and $v_{1 ; h}, \ldots, v_{4 ; h}$, respectively, in the manner of (22a). The vectors $\tilde{\mathbf{L}}^{T}=$ $\left[\tilde{\mathbf{L}}_{1,2}^{T}, \tilde{\mathbf{L}}_{2,4}^{T}, \tilde{\mathbf{L}}_{3,4}^{T}\right]$ and $\tilde{\mathbf{U}}^{T}=\left[\tilde{\mathbf{U}}_{1,2}^{T}, \tilde{\mathbf{U}}_{2,4}^{T}, \tilde{\mathbf{U}}_{3,4}^{T}\right]$ collect all coefficients used in the expansion of $\lambda_{1,2 ; h}, \lambda_{2,4 ; h}$, $\lambda_{3,4 ; h}$ and $\mu_{1,2 ; h}, \mu_{2,4 ; h}, \mu_{3,4 ; h}$, respectively, in the manner of (22c). Substitution of (24a)-(24d) into (23) finally yields the (saddle-point) linear system

$$
\begin{aligned}
& \text { Find }(\tilde{\mathbf{P}}, \tilde{\mathbf{L}}) \in \mathbb{C}^{\operatorname{dim} \mathcal{X}_{h}} \times \mathbb{C}^{\operatorname{dim} \mathcal{Y}_{h}} \text { such that } \\
& {\left[\begin{array}{ll}
\bar{A} & \overline{\boldsymbol{B}}^{T} \\
\overline{\mathbf{B}} &
\end{array}\right]\left[\begin{array}{l}
\tilde{\mathbf{P}} \\
\tilde{\mathbf{L}}
\end{array}\right]=\left[\begin{array}{l}
\boldsymbol{F} \\
\mathbf{0}
\end{array}\right]}
\end{aligned}
$$

We should emphasize here that we are solving in (25) for the phasor pressure fields, and hence as indicated, in general the matrices and solution vectors in (25) are complex-valued with real and imaginary parts.

\section{THE INF-SUP CONDITIONS}

We now turn to establish the tools for evaluating the well-posedness of the mixed method of finite spheres formulation. 


\subsection{Well-posedness of the discrete problems}

The bilinear forms $a$ in (19a) and $b$ in (19b) must be bounded, i.e., there must be positive constants $C_{1}$ and $C_{2}$ such that for any $(\mathbf{w}, \mathbf{v}) \in \mathcal{X} \times \mathcal{X}$, and for any $(\mathbf{v}, \boldsymbol{\mu}) \in \mathcal{X} \times \mathcal{Y}$,

$$
\begin{aligned}
& |a(\mathbf{w}, \mathbf{v})| \leq C_{1}\|\mathbf{w}\|_{\mathcal{X}}\|\mathbf{v}\|_{\mathcal{X}}, \\
& |b(\mathbf{v}, \boldsymbol{\mu})| \leq C_{2}\|\mathbf{v}\|_{\mathcal{X}}\|\boldsymbol{\mu}\|_{\mathcal{Y}},
\end{aligned}
$$

respectively. In the geometrical setting of Fig. $1,\|\cdot\|_{\mathcal{X}}$ and $\|\cdot\|_{\mathcal{Y}}$ refer to the norms of vectors in the spaces $\mathcal{X}$ and $\mathcal{Y}$ defined in (16a) and (16b), respectively (to be given below in Section 5.2). It can be shown that these bilinear forms are bounded. We then introduce the operator $B_{h}: \mathcal{X}_{h} \longrightarrow \mathcal{Y}_{h}^{\star}$ defined as: For any $\left(\mathbf{v}_{h}, \boldsymbol{\mu}_{h}\right) \in \mathcal{X}_{h} \times \mathcal{Y}_{h}$,

$$
\left\langle B_{h}\left(\mathbf{v}_{h}\right) \mid \boldsymbol{\mu}_{h}\right\rangle_{\mathcal{Y}_{h}^{\star}, \mathcal{Y}_{h}} \stackrel{\text { def }}{=} b\left(\mathbf{v}_{h}, \boldsymbol{\mu}_{h}\right),
$$

where $\mathcal{Y}_{h}^{\star}$ is the dual space of $\mathcal{Y}_{h}[12,13,41,42]$. The kernel (or null space) of $B_{h}$ is given by

$$
\operatorname{ker} B_{h} \stackrel{\text { def }}{=}\left\{\mathbf{v}_{h} \in \mathcal{X}_{h}: B_{h}\left(\mathbf{v}_{h}\right)=\mathbf{0} \text {, in } \mathcal{Y}_{h}^{\star}\right\} .
$$

The well-posedness of the finite-dimensional problem (23) is governed by the following theorem [12,13, 41]:

Theorem 5.1. Consider the finite-dimensional subspaces $\mathcal{X}_{h} \subset \mathcal{X}$ and $\mathcal{Y}_{h} \subset \mathcal{Y}$. Let $a: \mathcal{X} \times \mathcal{X} \longrightarrow \mathbb{C}$ and $b: \mathcal{X} \times \mathcal{Y} \longrightarrow \mathbb{C}$ be two bounded bilinear forms. The finite-dimensional saddle-point problem (23) is well-posed (i.e., its solution exists, is unique, and depends continuously on the data) if and only if the following two conditions are satisfied:

1. There is a constant $\alpha_{h}>0$ such that

$$
\inf _{\substack{\mathbf{w}_{h} \in \operatorname{ker} B_{h} \\ \mathbf{w}_{h} \neq \mathbf{0}}} \sup _{\substack{\mathbf{v}_{h} \in \operatorname{ker} B_{h} \\ \mathbf{v}_{h} \neq \mathbf{0}}} \frac{\left|a\left(\mathbf{w}_{h}, \mathbf{v}_{h}\right)\right|}{\left\|\mathbf{w}_{h}\right\|_{\mathcal{X}}\left\|\mathbf{v}_{h}\right\|_{\mathcal{X}}} \geq \alpha_{h} .
$$

2. There is a constant $\beta_{h}>0$ such that

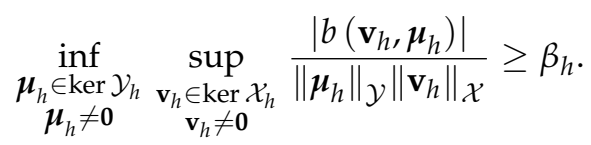

In the variational formulation of many problems in mechanics, the bilinear form $a$ has the property of coercivity, or ellipticity $[6,33,41,43]$, i.e., there is a positive constant $C$ such that for any $\mathbf{v} \in$ $\mathcal{X},|a(\mathbf{v}, \mathbf{v})| \geq C\|\mathbf{v}\|_{\mathcal{X}}^{2}$. Clearly if $a$ is coercive, then it satisfies (28a). In this case, only condition (28b) needs to be verified (e.g., the Stokes problem $[38,40]$ ). However, in the variational formulation of the Helmholtz equation considered here, the bilinear form $a$ in (19a) is not coercive, so that in this work both conditions must be verified. More details about problems with non-coercive bilinear forms (and other approaches to handle them, such as the Fredholm Alternative) can be found in the references [32,34,43-45].

\subsection{Norms of vectors}

In the example geometry of Fig. 1 , arbitrary vectors $\mathbf{v}=\left(v_{1}, v_{2}, v_{3}, v_{4}\right) \in \mathcal{X}$ and $\boldsymbol{\mu}=\left(\mu_{1,2}, \mu_{2,4}, \mu_{3,4}\right) \in$ $\mathcal{Y}$ have their norms given by

$$
\begin{aligned}
& \|\mathbf{v}\|_{\mathcal{X}}^{2} \stackrel{\text { def }}{=} \sum_{r=1}^{4}\left\|v_{r}\right\|_{H^{1}\left(\Omega_{r}\right)}^{2}, \\
& \|\boldsymbol{\mu}\|_{\mathcal{Y}}^{2} \stackrel{\text { def }}{=}\left\|\mu_{1,2}\right\|_{H^{-1 / 2}\left(\Gamma_{1,2}\right)}^{2}+\left\|\mu_{2,4}\right\|_{H^{-1 / 2}\left(\Gamma_{2,4}\right)}^{2}+\left\|\mu_{3,4}\right\|_{H^{-1 / 2}\left(\Gamma_{3,4}\right)}^{2},
\end{aligned}
$$


where the standard norms in the $H^{1}$ and $H^{-1 / 2}$ spaces are

$$
\begin{aligned}
& \left\|v_{r}\right\|_{H^{1}\left(\Omega_{r}\right)}^{2} \stackrel{\text { def }}{=} \int_{\Omega_{r}}\left(\nabla v_{r} \cdot \nabla v_{r}^{*}+v_{r} v_{r}^{*}\right) d \Omega, \\
& \left\|\mu_{1,2}\right\|_{H^{-1 / 2}\left(\Gamma_{1,2}\right)} \stackrel{\text { def }}{=} \sup _{\substack{t \in H^{1 / 2}\left(\Gamma_{1,2}\right) \\
t \neq 0}} \frac{\left|\left\langle\mu_{1,2} \mid t\right\rangle_{H^{1 / 2}\left(\Gamma_{1,2}\right)}\right|}{\|t\|_{H^{1 / 2}\left(\Gamma_{1,2}\right)}} .
\end{aligned}
$$

The norm $\|\cdot\|_{H^{-1 / 2}\left(\Gamma_{1,2}\right)}$ defined in (29d) is a dual norm (since $H^{-1 / 2}$ is the dual space of $H^{1 / 2}$ ). The norm $\|\cdot\|_{H^{1 / 2}\left(\Gamma_{1,2}\right)}$ is given in Appendix B. The terms $\left\|\mu_{2,4}\right\|_{H^{-1 / 2}\left(\Gamma_{2,4}\right)}$ and $\left\|\mu_{3,4}\right\|_{H^{-1 / 2}\left(\Gamma_{3,4}\right)}$ are defined likewise. The finite-dimensional subspaces $\mathcal{X}_{h} \subset \mathcal{X}$ and $\mathcal{Y}_{h} \subset \mathcal{Y}$ introduced in Section 4 inherit the norms in (29a) and (29b), respectively.

Given a meshfree setting characterized by the discretization length $h$ (see [10]), we can guarantee the well-posedness of our discrete problem (23) if we show that (28a) and (28b) hold true. However, because of the complications involved due to the use of the dual norm $H^{-1 / 2}$, as indicated in (29b) and (29d), we derive new inf-sup conditions simpler than (28a) and (28b), but which at the same time are sufficient to imply (28a) and (28b), i.e., we shall use stronger conditions.

\section{THE FIRST INF-SUP CONDITION}

In this section we discuss an inf-sup condition which is stronger than condition (28a).

\subsection{Stronger condition}

Considering the bilinear form $a: \mathcal{X} \times \mathcal{X} \longrightarrow \mathbb{C}$ from (19a), since $\operatorname{Re}\left\{a\left(\mathbf{w}_{h}, \mathbf{v}_{h}\right)\right\} \leq\left|a\left(\mathbf{w}_{h}, \mathbf{v}_{h}\right)\right|$ for any $\mathbf{w}_{h}, \mathbf{v}_{h} \in \mathcal{X}_{h} \backslash\{\mathbf{0}\}$, we shall use:

There is a constant $\alpha_{h}>0$ such that

$$
\inf _{\substack{\mathbf{w}_{h} \in \operatorname{ker} \\ \mathbf{w}_{h} \neq \mathbf{0}}} \sup _{\substack{\mathbf{v}_{h} \in \operatorname{ker} \\ \mathbf{v}_{h} \neq \mathbf{0}}} \frac{\operatorname{Re}\left\{a\left(\mathbf{w}_{h}, \mathbf{v}_{h}\right)\right\}}{\left\|\mathbf{w}_{h}\right\|_{\mathcal{X}}\left\|\mathbf{v}_{h}\right\|_{\mathcal{X}}} \geq \alpha_{h} .
$$

\subsection{Stronger condition, linear-algebraic aspects}

Given an arbitrary vector $\mathbf{v}_{h}=\left(v_{1 ; h}, v_{2 ; h}, v_{3 ; h}, v_{4 ; h}\right) \in \mathcal{X}_{h}$, if we use the expansion (22a) for $r=$ $1, \ldots, 4$ in $(29 \mathrm{c})$, it is straightforward to express (29a) in matrix form as

$$
\left\|\mathbf{v}_{h}\right\|_{\mathcal{X}}^{2}=\sum_{r=1}^{4} \tilde{\mathbf{V}}_{r}^{\dagger} \overline{\boldsymbol{S}}_{r} \tilde{\mathbf{V}}_{r}=\underbrace{\left[\begin{array}{c}
\tilde{\mathbf{V}}_{1} \\
\tilde{\mathbf{V}}_{2} \\
\tilde{\mathbf{V}}_{3} \\
\tilde{\mathbf{V}}_{4}
\end{array}\right]^{\dagger}}_{\tilde{\mathbf{V}}} \underbrace{\left[\begin{array}{cccc}
\overline{\boldsymbol{S}}_{1} & & & \\
& \overline{\boldsymbol{S}}_{2} & & \\
& & \overline{\boldsymbol{S}}_{3} & \\
& & & \overline{\boldsymbol{S}}_{4}
\end{array}\right]}_{\overline{\boldsymbol{S}}} \underbrace{\left[\begin{array}{c}
\tilde{\mathbf{V}}_{1} \\
\tilde{\mathbf{V}}_{2} \\
\tilde{\mathbf{V}}_{3} \\
\tilde{\mathbf{V}}_{4}
\end{array}\right]}_{\tilde{\mathbf{V}}}=\tilde{\mathbf{V}}^{\dagger} \overline{\boldsymbol{S}} \tilde{\mathbf{V}},
$$

where $\tilde{\mathbf{V}}_{r}^{\dagger}$ is the conjugate transposed version of the coefficient vector $\tilde{\mathbf{V}}_{r}$. The symmetric matrices $\overline{\boldsymbol{S}}_{r}$ belong to $\mathbb{R}^{\operatorname{dim} \mathcal{M}\left(\Omega_{r}\right) \times \operatorname{dim} \mathcal{M}\left(\Omega_{r}\right)}$, and the matrix $\bar{S}$ (also symmetric) belongs to $\mathbb{R}^{\operatorname{dim} \mathcal{X}_{h} \times \operatorname{dim} \mathcal{X}_{h}}$.

The kernel of the matrix $\overline{\boldsymbol{B}}$ introduced in (24b) is $\operatorname{ker} \overline{\boldsymbol{B}} \stackrel{\text { def }}{=}\left\{\tilde{\mathbf{V}} \in \mathbb{C}^{\operatorname{dim} \mathcal{X}_{h}}: \overline{\boldsymbol{B}} \tilde{\mathbf{V}}=\mathbf{0}\right\}$, where $\mathbf{0}$ is the zero vector of length $\operatorname{dim} \mathcal{Y}_{h}$. There is a bijection between $\operatorname{ker} B_{h}$ in $(27 \mathrm{~b})$ and $\operatorname{ker} \overline{\boldsymbol{B}}$, thanks to the isomorphism between $\mathcal{X}_{h}$ and $\mathbb{C}^{\operatorname{dim} \mathcal{X}_{h}}$. Using the matrix representation as in (24a) and (30b), condition (30a) becomes equivalent to:

There is a constant $\alpha_{h}>0$ such that

$$
\inf _{\substack{\tilde{\mathbf{W}} \in \operatorname{ker} \\ \tilde{\mathbf{W}} \neq \mathbf{0}}} \sup _{\substack{\tilde{\mathbf{V}} \in \operatorname{Ver} \\ \tilde{\mathbf{V}} \neq \mathbf{0}}} \frac{\operatorname{Re}\left\{\tilde{\mathbf{V}}^{T} \bar{A} \tilde{\mathbf{V}}\right\}}{\sqrt{\tilde{\mathbf{W}}^{+} \bar{S} \tilde{\mathbf{W}}} \sqrt{\tilde{\mathbf{V}}^{+} \overline{\boldsymbol{S}} \tilde{\mathbf{V}}}} \geq \alpha_{h} .
$$

We now find a basis for $\operatorname{ker} \overline{\boldsymbol{B}}$. Suppose the dimension of $\operatorname{ker} \overline{\boldsymbol{B}}$ is $K$ i.e., $\operatorname{dim} \operatorname{ker} \overline{\boldsymbol{B}}=K$. We look for a linearly independent set of $K$ vectors $\psi_{k} \in \mathbb{C}^{\operatorname{dim} \mathcal{X}_{h}}$ such that $\operatorname{ker} \overline{\boldsymbol{B}}=\operatorname{span}\left\{\psi_{1}, \ldots, \psi_{K}\right\}$. These 
basis vectors are arranged into a matrix $\bar{\Psi} \in \mathbb{C}^{\operatorname{dim} \mathcal{X}_{h} \times K}$ as $\overline{\mathbf{\Psi}}=\left[\psi_{1}, \ldots, \psi_{K}\right]$. The map $\tilde{\mathbf{Z}} \longmapsto \overline{\mathbf{\Psi}} \tilde{\mathbf{Z}}$ is an isomorphism between $\mathbb{C}^{K}$ and $\operatorname{ker} \bar{B}$, so that condition (30c) becomes equivalent to:

There is a constant $\alpha_{h}>0$ such that

$$
\inf _{\substack{\tilde{\mathbf{D}} \in \mathbb{C}^{K} \\ \tilde{\mathbf{D}} \neq \mathbf{0}}} \sup _{\substack{\tilde{\mathbf{C}} \in \mathbb{C}^{K} \\ \tilde{\mathbf{C}} \neq \mathbf{0}}} \frac{\operatorname{Re}\left\{\tilde{\mathbf{C}}^{T} \overline{\mathbf{\Psi}}^{T} \bar{A} \overline{\mathbf{\Psi}} \tilde{\mathbf{D}}\right\}}{\sqrt{\tilde{\mathbf{D}}^{+} \bar{\Psi}^{\dagger} \bar{S} \overline{\mathbf{\Psi}} \tilde{\mathbf{D}}} \sqrt{\tilde{\mathbf{C}}^{\dagger} \bar{\Psi}^{\dagger} \overline{\boldsymbol{S}} \overline{\mathbf{\Psi}} \tilde{\mathbf{C}}}} \geq \alpha_{h} .
$$

If we let $\overline{\boldsymbol{I}}_{K} \in \mathbb{R}^{K \times K}$ denote the identity matrix of size $K \times K$, then the natural isomorphism between $\mathbb{R}^{2 K}$ and $\mathbb{C}^{K}$ can be represented by the matrix $\overline{\mathbf{J}}_{K} \stackrel{\text { def }}{=}\left[\overline{\mathbf{I}}_{K}, j \overline{\mathbf{I}}_{K}\right] \in \mathbb{C}^{K \times 2 K}$, via the map $\tilde{\mathfrak{w}} \longmapsto \overline{\mathbf{J}}_{K} \tilde{\mathfrak{w}}$ (this transformation takes any vector of real numbers of size $2 K$ and uniquely associates with it a vector of complex numbers of size $K$ ). In the rest of this text, real-valued vectors of coefficients will be written using lowercase bold German letters with a tilde, such as $\tilde{\mathfrak{w}}$. Condition (30d) becomes equivalent to:

There is a constant $\alpha_{h}>0$ such that

$$
\inf _{\substack{\tilde{\mathfrak{w}} \in \mathbb{R}^{2 K} \\ \mathfrak{\mathfrak { w }} \neq \mathbf{0}}} \sup _{\substack{\mathfrak{\mathfrak { v }} \in \mathbb{R}^{2 K} \\ \mathfrak{\mathfrak { v }} \neq \mathbf{0}}} \frac{\operatorname{Re}\left\{\tilde{\mathfrak{v}}^{T} \overline{\mathbf{J}}_{K}^{T} \bar{\Psi}^{T} \bar{A} \bar{\Psi} \overline{\mathbf{J}}_{K} \tilde{\mathfrak{w}}\right\}}{\sqrt{\tilde{\mathfrak{w}}^{+} \overline{\mathbf{J}}_{K}^{+} \bar{\Psi}^{+} \bar{S} \bar{\Psi} \overline{\mathbf{J}}_{K} \tilde{\mathfrak{w}}} \sqrt{\tilde{\mathfrak{v}}^{+} \overline{\mathbf{J}}_{K}^{+} \bar{\Psi}^{+} \bar{S} \bar{\Psi} \overline{\mathbf{J}}_{K} \tilde{\mathfrak{v}}}} \geq \alpha_{h} .
$$

The vectors $\tilde{\mathfrak{w}}$ and $\tilde{\mathfrak{v}}$ are real-valued, which implies that $\tilde{\mathfrak{w}}^{\dagger}=\tilde{\mathfrak{w}}^{T}$ and $\tilde{\mathfrak{v}}^{\dagger}=\tilde{\mathfrak{v}}^{T}$. The numerator in (30e) is rewritten as $\tilde{\mathfrak{v}}^{T} \overline{\mathbb{A}} \tilde{\mathfrak{w}}$, where the matrix $\overline{\mathbb{A}} \stackrel{\text { def }}{=} \operatorname{Re}\left\{\overline{\mathbf{J}}_{K}^{T} \overline{\mathbf{\Psi}}^{T} \bar{A} \bar{\Psi} \overline{\mathbf{J}}_{K}\right\}$ belongs to $\mathbb{R}^{2 K \times 2 K}$. It is a symmetric matrix, since the matrix $\bar{A}$ in (24a) is symmetric. The term inside the first square root in the denominator of (30e) is rewritten as $\tilde{\mathfrak{w}}^{T} \overline{\mathbb{D}} \tilde{\mathfrak{w}}$, where the symmetric matrix $\overline{\mathbb{D}} \stackrel{\text { def }}{=} \operatorname{Re}\left\{\overline{\mathbf{J}}_{K}^{\dagger} \overline{\mathbf{\Psi}}^{\dagger} \bar{S} \bar{\Psi} \overline{\mathbf{J}}_{K}\right\}$ belongs to $\mathbb{R}^{2 K \times 2 K}$ (the matrix $\bar{S}$ in (30b) is real and symmetric, and it can be verified that the imaginary part of $\overline{\mathbf{J}}_{K}^{\dagger} \overline{\mathbf{\Psi}}^{\dagger} \overline{\boldsymbol{S}} \overline{\mathbf{\Psi}} \overline{\mathbf{J}}_{K}$ satisfies $\tilde{\mathfrak{w}}^{\dagger} \operatorname{Im}\left\{\overline{\mathbf{J}}_{K}^{\dagger} \overline{\mathbf{\Psi}}^{\dagger} \overline{\boldsymbol{S}} \bar{\Psi} \overline{\mathbf{J}}_{K}\right\} \tilde{\mathfrak{w}}=0$ ). The second term in the denominator of (30e) is treated likewise. The inf-sup condition (30e) assumes its final form, becoming equivalent to:

There is a constant $\alpha_{h}>0$ such that

$$
\inf _{\substack{\tilde{\mathfrak{w}} \in \mathbb{R}^{2 K} \\ \mathfrak{w} \neq 0}} \sup _{\substack{\mathfrak{\mathfrak { v }} \in \mathbb{R}^{2 K} \\ \mathfrak{\mathfrak { v }} \neq \mathbf{0}}} \frac{\tilde{\mathfrak{w}}^{T} \overline{\mathbb{A}} \tilde{\mathfrak{v}}}{\sqrt{\tilde{\mathfrak{w}}^{T} \overline{\mathbb{D}} \tilde{\mathfrak{w}}} \sqrt{\tilde{\mathfrak{v}}^{T} \overline{\mathbf{D}} \tilde{\mathfrak{v}}}} \geq \alpha_{h}
$$

where we used the fact that $\tilde{\mathfrak{v}}^{T} \overline{\mathbb{A}} \tilde{\mathfrak{w}}=\tilde{\mathfrak{w}}^{T} \overline{\mathbb{A}}^{T} \tilde{\mathfrak{v}}=\tilde{\mathfrak{w}}^{T} \overline{\mathbb{A}} \tilde{\mathfrak{v}}$, since $\overline{\mathbb{A}}$ is symmetric. Condition (31) is written entirely in terms of real-valued vectors and matrices, despite the original function spaces used in the scattering problem being complex-valued in nature.

\section{THE SECOND INF-SUP CONDITION}

Our goal is now to discuss an inf-sup condition which is stronger than condition (28b).

\subsection{Stronger condition}

Since for any $\boldsymbol{\mu}_{h} \in \mathcal{Y}_{h} \backslash\{\boldsymbol{0}\}$ and $\mathbf{v}_{h} \in \mathcal{X}_{h} \backslash\{\mathbf{0}\}$ we have that $\operatorname{Re}\left\{b\left(\mathbf{v}_{h}, \boldsymbol{\mu}_{h}\right)\right\} \leq\left|b\left(\mathbf{v}_{h}, \boldsymbol{\mu}_{h}\right)\right|$, we use the following stronger condition:

There is a constant $\beta_{h}>0$ such that

$$
\inf _{\substack{\boldsymbol{\mu}_{h} \in \mathcal{Y}_{h} \\ \boldsymbol{\mu}_{h} \neq \mathbf{0}}} \sup _{\substack{\mathbf{v}_{h} \in \mathcal{X}_{h} \\ \mathbf{v}_{h} \neq \mathbf{0}}} \frac{\operatorname{Re}\left\{b\left(\mathbf{v}_{h}, \boldsymbol{\mu}_{h}\right)\right\}}{\left\|\boldsymbol{\mu}_{h}\right\|_{\mathcal{Y}}\left\|\mathbf{v}_{h}\right\|_{\mathcal{X}}} \geq \beta_{h} .
$$

To evaluate this condition we need the following result:

Theorem 7.1. Let $\Sigma$ be a simple (i.e., which does not cross itself), closed (endpoints coincide), and Lipschitz continuous curve in $\mathbb{R}^{2}$. Let $\Sigma^{o}$ denote the region of the plane interior to the curve $\Sigma$. Then, there is a positive constant $C_{\Sigma^{o}}$ (which depends only on the geometry of $\Sigma^{o}$ ) such that for any $\theta \in H^{-1 / 2}(\Sigma)$,

1. There is a unique function $f_{\theta} \in H^{1}\left(\Sigma^{o}\right)$ such that

$$
\|\theta\|_{H^{-1 / 2}(\Sigma)} \leq C_{\Sigma^{o}}\left\|f_{\theta}\right\|_{H^{1}\left(\Sigma^{o}\right)} .
$$


2. This function $f_{\theta}$ satisfies the variational equality:

$$
\int_{\Sigma^{o}}\left(\nabla w \cdot \nabla f_{\theta}+w f_{\theta}\right) d \Omega=\left\langle\theta \mid \gamma_{\Sigma}(w)\right\rangle_{H^{1 / 2}(\Sigma)}, \text { for any } w \in H^{1}\left(\Sigma^{o}\right) .
$$

This result (proved in Appendix D) is fundamental since it releases us from the task of working with the dual norm $H^{-1 / 2}$ (see (29b) and (29d)). In (33b), $\gamma_{\Sigma}(w)$ is the trace of $w$ along $\Sigma$. In the geometrical setting of Fig. 1 , let $\Gamma_{1,2}^{o}, \Gamma_{2,4}^{o}$, and $\Gamma_{3,4}^{o}$ denote the regions of the plane interior to the closed curves (interfaces) $\Gamma_{1,2}, \Gamma_{2,4}$, and $\Gamma_{3,4}$, respectively. We form the space

$$
\mathcal{Z} \stackrel{\text { def }}{=} H^{1}\left(\Gamma_{1,2}^{o}\right) \times H^{1}\left(\Gamma_{2,4}^{o}\right) \times H^{1}\left(\Gamma_{3,4}^{o}\right) .
$$

Arbitrary elements from $\mathcal{Z}$ can be represented by vectors as $\mathbf{z}=\left(z_{1,2}, z_{2,4}, z_{3,4}\right)$. We equip $\mathcal{Z}$ with the following norm: For any $\mathbf{z} \in \mathcal{Z}$,

$$
\|\mathbf{z}\|_{\mathcal{Z}}^{2} \stackrel{\text { def }}{=}\left\|z_{1,2}\right\|_{H^{1}\left(\Gamma_{1,2}^{o}\right)}^{2}+\left\|z_{2,4}\right\|_{H^{1}\left(\Gamma_{2,4}^{o}\right)}^{2}+\left\|z_{3,4}\right\|_{H^{1}\left(\Gamma_{3,4}^{o}\right)}^{2} .
$$

We next consider an arbitrary vector of discretized functionals $\mu_{h}=\left(\mu_{1,2 ; h}, \mu_{2,4 ; h}, \mu_{3,4 ; h}\right) \in \mathcal{Y}_{h}$, see Section 4. Application of Theorem 7.1 to each component gives the existence of three positive constants $C_{\Gamma_{1,2}^{o}}, C_{\Gamma_{2,4}^{o}}$, and $C_{\Gamma_{3,4}^{o}}$ such that

$$
\begin{aligned}
&\left\|\mu_{1,2 ; h}\right\|_{H^{-1 / 2}\left(\Gamma_{1,2}\right)} \leq C_{\Gamma_{1,2}^{o}}\left\|f_{\mu_{1,2 ; h}}\right\|_{H^{1}\left(\Gamma_{1,2}^{o}\right)^{\prime}}, \\
&\left\|\mu_{2,4 ; h}\right\|_{H^{-1 / 2}\left(\Gamma_{2,4}\right)} \leq C_{\Gamma_{2,4}^{o}}\left\|f_{\mu_{2,4 ; h}}\right\|_{H^{1}\left(\Gamma_{2,4}^{o}\right)^{\prime}}, \\
&\left\|\mu_{3,4 ; h}\right\|_{H^{-1 / 2}\left(\Gamma_{3,4}\right)} \leq C_{\Gamma_{3,4}^{o}}\left\|f_{\mu_{3,4 ; h}}\right\|_{H^{1}\left(\Gamma_{3,4}^{o}\right)^{\prime}}
\end{aligned}
$$

where the functions $f_{\mu_{1,2 ; h}} \in H^{1}\left(\Gamma_{1,2}^{o}\right), f_{\mu_{2,4 ; h}} \in H^{1}\left(\Gamma_{2,4}^{o}\right)$, and $f_{\mu_{3,4 ; h}} \in H^{1}\left(\Gamma_{3,4}^{o}\right)$ are solutions to the problems

$$
\begin{aligned}
& \int_{\Gamma_{1,2}^{o}}\left(\nabla w_{1,2} \cdot \nabla f_{\mu_{1,2 ; h}}+w_{1,2} f_{\mu_{1,2 ; h}}\right) d \Omega=\left\langle\mu_{1,2 ; h} \mid \gamma_{\Gamma_{1,2}}\left(w_{1,2}\right)\right\rangle_{H^{1 / 2}\left(\Gamma_{1,2}\right)}, \text { for any } w_{1,2} \in H^{1}\left(\Gamma_{1,2}^{o}\right), \\
& \int_{\Gamma_{2,4}^{o}}\left(\nabla w_{2,4} \cdot \nabla f_{\mu_{2,4, h}}+w_{2,4} f_{\mu_{2,4 ; h}}\right) d \Omega=\left\langle\mu_{2,4 ; h} \mid \gamma_{\Gamma_{2,4}}\left(w_{2,4}\right)\right\rangle_{H^{1 / 2}\left(\Gamma_{2,4}\right)}, \text { for any } w_{2,4} \in H^{1}\left(\Gamma_{2,4}^{o}\right), \\
& \int_{\Gamma_{3,4}^{o}}\left(\nabla w_{3,4} \cdot \nabla f_{\mu_{3,4 ; h}}+w_{3,4} f_{\mu_{3,4 ; h}}\right) d \Omega=\left\langle\mu_{3,4 ; h} \mid \gamma_{\Gamma_{3,4}}\left(w_{3,4}\right)\right\rangle_{H^{1 / 2}\left(\Gamma_{3,4}\right)}, \text { for any } w_{3,4} \in H^{1}\left(\Gamma_{3,4}^{o}\right),
\end{aligned}
$$

respectively. Using these functions, we define the vector $\mathbf{f}_{\boldsymbol{\mu}_{h}} \stackrel{\text { def }}{=}\left(f_{\mu_{1,2 ; h},} f_{\mu_{2,4 ; h},} f_{\mu_{3,4 ; h}}\right) \in \mathcal{Z}$. Based on (29b) and (34b), the three inequalities in (34c) allow us to conclude that there is a positive constant $C$ (independent of $\boldsymbol{\mu}_{h}$ ) such that

$$
\left\|\boldsymbol{\mu}_{h}\right\|_{\mathcal{Y}} \leq C\left\|\mathbf{f}_{\boldsymbol{\mu}_{h}}\right\|_{\mathcal{Z}}
$$

We can now introduce the following inf-sup condition:

There is a constant $\beta_{h}>0$ such that

$$
\inf _{\substack{\boldsymbol{\mu}_{h} \in \mathcal{Y}_{h} \\ \boldsymbol{\mu}_{h} \neq \mathbf{0}}} \sup _{\substack{\mathbf{v}_{h} \in \mathcal{X}_{h} \\ \mathbf{v}_{h} \neq \mathbf{0}}} \frac{\operatorname{Re}\left\{b\left(\mathbf{v}_{h}, \boldsymbol{\mu}_{h}\right)\right\}}{\left\|\mathbf{f}_{\boldsymbol{\mu}_{h}}\right\|\left\|_{\mathcal{Z}}\right\| \mathbf{v}_{h} \|_{\mathcal{X}}} \geq \beta_{h} .
$$

Since we have (34e), it follows that (35) implies (32), i.e., it is 'stronger' than (32). The inf-sup condition (35) does not involve the norm in the space $\mathcal{Y}$ (given in terms of $H^{-1 / 2}$ norms, see (29b) and (29d)) as in (32), and hence we shall use it. However, we must solve problems (34d) in order to find the functions $f_{\mu_{1,2 ; h},} f_{\mu_{2,4 ;},}$, and $f_{\mu_{3,4 ;}}$. We use meshfree finite-dimensional subspaces of $H^{1}\left(\Gamma_{1,2}^{o}\right), H^{1}\left(\Gamma_{2,4}^{o}\right)$, and $H^{1}\left(\Gamma_{3,4}^{o}\right)$, and solve for approximations of these functions within these spaces. 


\subsection{Meshfree finite dimensional subspaces for the interior regions}

For each interior region $\Gamma_{1,2}^{o}, \Gamma_{2,4}^{o}$, and $\Gamma_{3,4}^{o}$, we construct meshfree spaces $\mathcal{M}\left(\Gamma_{1,2}^{o}\right), \mathcal{M}\left(\Gamma_{2,4}^{o}\right)$, and $\mathcal{M}\left(\Gamma_{3,4}^{o}\right)$, respectively, using the process outlined in Section 4 . Arbitrary functions $z_{1,2} \in H^{1}\left(\Gamma_{1,2}^{o}\right), z_{2,4} \in$ $H^{1}\left(\Gamma_{2,4}^{o}\right)$ and $z_{3,4} \in H^{1}\left(\Gamma_{3,4}^{o}\right)$ together with their traces can be approximated by their discretized equivalents as

$$
\begin{gathered}
z_{1,2 ; h}=\mathbf{E}_{1,2}^{T} \tilde{\mathbf{Z}}_{1,2}, \quad z_{2,4 ; h}=\mathbf{E}_{2,4}^{T} \tilde{\mathbf{Z}}_{2,4}, \quad z_{3,4 ; h}=\mathbf{E}_{3,4}^{T} \tilde{\mathbf{Z}}_{3,4}, \\
\gamma_{\Gamma_{1,2}}\left(z_{1, z ; h}\right)=\mathbf{R}_{1,2}^{T} \tilde{\mathbf{Z}}_{1,2}, \quad \gamma_{\Gamma_{2,4}}\left(z_{2,4 ; h}\right)=\mathbf{R}_{2,4}^{T} \tilde{\mathbf{Z}}_{2,4}, \quad \gamma_{\Gamma_{3,4}}\left(z_{3,4 ; h}\right)=\mathbf{R}_{3,4}^{T} \tilde{\mathbf{Z}}_{3,4},
\end{gathered}
$$

where $\mathbf{E}_{1,2}: \Gamma_{1,2}^{o} \longrightarrow \mathbb{R}^{\operatorname{dim} \mathcal{M}\left(\Gamma_{1,2}^{o}\right)}$ is a vector function collecting all basis functions defined on $\Gamma_{1,2}^{o}$, and $\mathbf{R}_{1,2}: \Gamma_{1,2} \longrightarrow \mathbb{R}^{\operatorname{dim} \mathcal{M}\left(\Gamma_{1,2}^{o}\right)}$ is a vector function which collects the traces of all basis functions along the

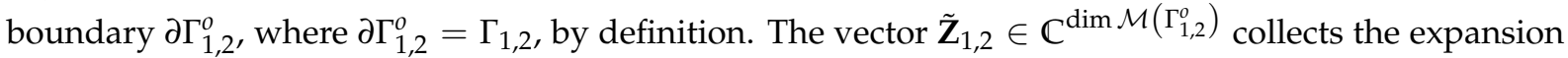
coefficients. The same holds for the expansions of $z_{2,4 ; h}$ and $z_{3,4 ; h}$ above.

Note that the closed curve $\Gamma_{1,2}$ does not encircle any other curve, because region $\Omega_{1}$ is simplyconnected. It follows that $\Gamma_{1,2}^{o}=\Omega_{1}$. Hence to approximate $z_{1,2} \in H^{1}\left(\Gamma_{1,2}^{o}\right)$ we use the same set of basis functions as that used to approximate $v_{1} \in H^{1}\left(\Omega_{1}\right)$, see Section 4, i.e., in (36a) and (36b) we can define $\mathbf{E}_{1,2} \stackrel{\text { def }}{=} \mathbf{H}_{1}$ and $\mathbf{R}_{1,2} \stackrel{\text { def }}{=} \mathbf{T}_{1}$, where $\mathbf{H}_{1}$ and $\mathbf{T}_{1}$ have been introduced in (22a) and (22b), respectively. The same holds for the closed curve $\Gamma_{3,4}$, since $\Omega_{3}$ is simply-connected and $\Gamma_{3,4}^{o}=\Omega_{3}$. We thus define $\mathbf{E}_{3,4} \stackrel{\text { def }}{=} \mathbf{H}_{3}$ and $\mathbf{R}_{3,4} \stackrel{\text { def }}{=} \mathbf{T}_{3}$. In the case of curve $\Gamma_{2,4}$, its interior $\Gamma_{2,4}^{o}$ does not coincide with $\Omega_{2}$ (see Fig. 4). A separate set of basis functions must be set up for approximating functions defined on $\Gamma_{2,4}^{o}$, together with their traces along $\Gamma_{2,4}$. The vector function $\mathbf{E}_{2,4}$ in (36a) differs from $\mathbf{H}_{2}$ in (22a), and $\mathbf{R}_{2,4}$ in (36b) differs from $\mathbf{T}_{2}$ in (22b).

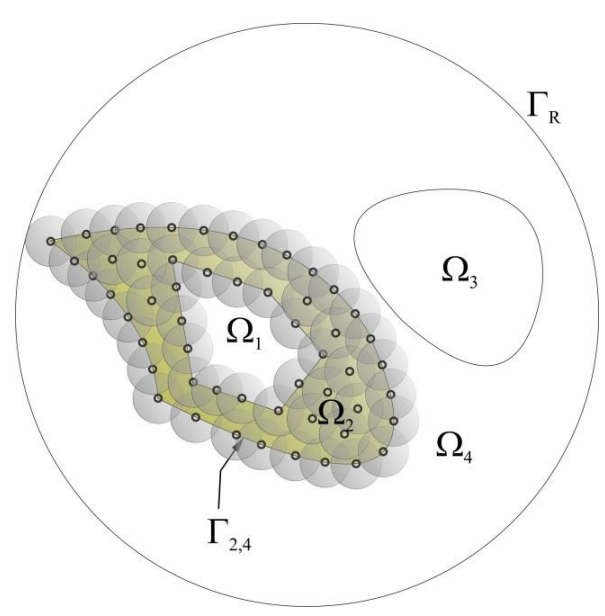

(a)

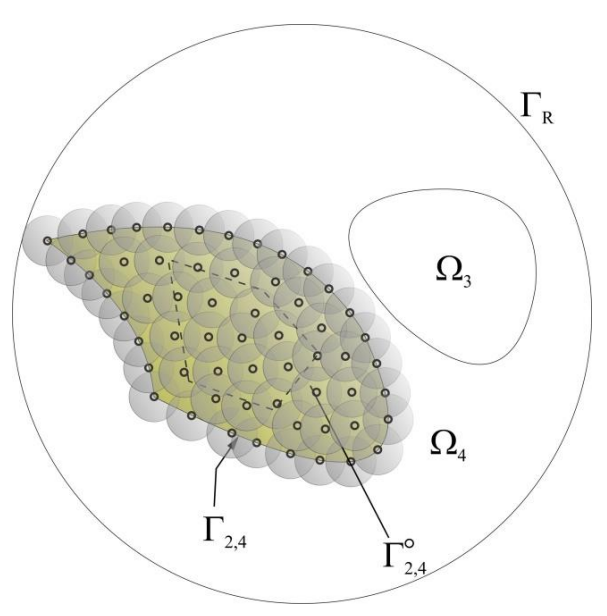

(b)

Fig. 4. Consider the interface $\Gamma_{2,4}$, between regions $\Omega_{2}$ and $\Omega_{4}$. The region $\Omega_{2}$ is not simply-connected (due to the hole left by $\left.\Omega_{1}\right)$. Consequently, the region interior to the curve $\Gamma_{2,4}$, denoted by $\Gamma_{2,4}^{o}$, is distinct from $\Omega_{2}$. This originates two different sets of basis functions. (a) Balls in a finite sphere system for approximating functions defined on $\Omega_{2}$. Their union must cover the closure of $\Omega_{2}$ (b) Balls in a finite sphere system for approximating functions defined on $\Gamma_{2,4}^{o}$. Their union must cover the closure of $\Gamma_{2,4}^{o}$

Once the meshfree spaces $\mathcal{M}\left(\Gamma_{1,2}^{o}\right), \mathcal{M}\left(\Gamma_{2,4}^{o}\right)$, and $\mathcal{M}\left(\Gamma_{3,4}^{o}\right)$ have been constructed, we introduce the finite dimensional subspace $\mathcal{Z}_{h}$ of $\mathcal{Z}$ in (34a) as:

$$
\mathcal{Z}_{h} \stackrel{\text { def }}{=} \mathcal{M}\left(\Gamma_{1,2}^{o}\right) \times \mathcal{M}\left(\Gamma_{2,4}^{o}\right) \times \mathcal{M}\left(\Gamma_{3,4}^{o}\right) .
$$

The norm in $\mathcal{Z}_{h}$ is that inherited from $\mathcal{Z}$ in (34b). The dimension of $\mathcal{Z}_{h}$ is given by the sum of the dimensions of $\mathcal{M}\left(\Gamma_{1,2}^{o}\right), \mathcal{M}\left(\Gamma_{2,4}^{o}\right)$, and $\mathcal{M}\left(\Gamma_{3,4}^{o}\right)$. 


\subsection{Stronger condition, linear-algebraic aspects}

We now turn our attention to condition (35). Arbitrary vectors of functionals $\mu_{h} \in \mathcal{Y}_{h}$ can be written as $\mu_{h}=\left(\mu_{1,2 ; h}, \mu_{2,4 ; h}, \mu_{3,4 ; h}\right)$, see Section 4 . The arbitrary functionals $\mu_{1,2 ; h}, \mu_{2,4 ; h}$, and $\mu_{3,4 ; h}$ determine the functions $f_{\mu_{1,2 ; h}}, f_{\mu_{2,4 ;}}$, and $f_{\mu_{3,4 ;}}$, respectively, via the variational problems in (34d), see Section 7.1. In other words, the vector $\boldsymbol{\mu}_{h}$ determines the vector $\mathbf{f}_{\boldsymbol{\mu}_{h}} \stackrel{\text { def }}{=}\left(f_{\mu_{1,2 ; h}}, f_{\mu_{2,4 ; h}} f_{\mu_{3,4 ; h}}\right)$. We rewrite (35) as:

There is a constant $\beta_{h}>0$ such that

$$
\inf _{\boldsymbol{\mu}_{h}=\left(\mu_{1,2 ; h}, \mu_{2,4 ; h}, \mu_{3,4 ; h}\right) \in \mathcal{Y}_{h}} \sup _{\substack{\mathbf{v}_{h} \in \mathcal{X}_{h} \neq \mathbf{0} \\ \mathbf{v}_{h} \neq \mathbf{0}}} \frac{\operatorname{Re}\left\{b\left(\mathbf{v}_{h}, \boldsymbol{\mu}_{h}\right)\right\}}{\left\|\left(f_{\mu_{1,2 ; h},} f_{\mu_{2,4 ; h},} f_{\mu_{3,4 ; h}}\right)\right\|_{\mathcal{Z}}\left\|\mathbf{v}_{h}\right\|_{\mathcal{X}}} \geq \beta_{h} .
$$

Let the coefficient vectors $\tilde{\mathbf{V}} \in \mathbb{C}^{\operatorname{dim} \mathcal{X}_{h}}$ and $\tilde{\mathbf{U}} \in \mathbb{C}^{\operatorname{dim} \mathcal{Y}_{h}}$ be arbitrary. These coefficient vectors can be broken into subvectors as $\tilde{\mathbf{V}}^{T}=\left[\tilde{\mathbf{V}}_{1}^{T}, \tilde{\mathbf{V}}_{2}^{T}, \tilde{\mathbf{V}}_{3}^{T}, \tilde{\mathbf{V}}_{4}^{T}\right]$ and $\tilde{\mathbf{U}}^{T}=\left[\tilde{\mathbf{U}}_{1,2}^{T}, \tilde{\mathbf{U}}_{2,4}^{T}, \tilde{\mathbf{U}}_{3,4}^{T}\right]$, see Section 4 . The vector $\mathbf{v}_{h} \in \mathcal{X}_{h}$ in (37) collects the four discrete fields as $\mathbf{v}_{h}=\left(v_{1 ; h}, v_{2 ; h}, v_{3 ; h}, v_{4 ; h}\right)$, see Section 4 . The component $v_{1 ; h}$ is determined by the coefficient subvector $\tilde{\mathbf{V}}_{1}$ as in (22a), and likewise for the other components. It follows that the coefficient vector $\tilde{\mathbf{V}}$ ultimately determines the vector $\mathbf{v}_{h}$. In the functionals, the components $\mu_{1,2 ; h}, \mu_{2,4 ; h}$, and $\mu_{3,4 ; h}$ are determined by the coefficient subvectors $\tilde{\mathbf{U}}_{1,2}, \tilde{\mathbf{U}}_{2,4}$, and $\tilde{\mathbf{U}}_{3,4}$, respectively, as in (22c). It follows that the coefficient vector $\tilde{\mathbf{U}}$ ultimately determines the vector $\boldsymbol{\mu}_{h}$. The numerator in (37) is therefore rewritten as $\operatorname{Re}\left\{b\left(\mathbf{v}_{h}, \boldsymbol{\mu}_{h}\right)\right\}=\operatorname{Re}\left\{\tilde{\mathbf{U}}^{T} \overline{\boldsymbol{B}} \tilde{\mathbf{V}}\right\}$, using (24d). The second term in the denominator of (37) is written as $\left\|\mathbf{v}_{h}\right\|_{x}=\sqrt{\tilde{\mathbf{V}}^{\dagger} \bar{S} \tilde{\mathbf{V}}}$, using (30b). Condition (37) becomes:

There is a constant $\beta_{h}>0$ such that

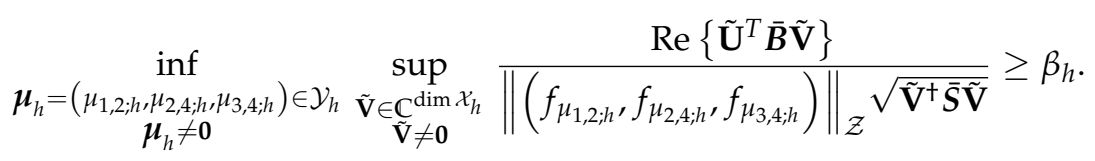

In order to find $f_{\mu_{1,2 ; h}}$, we use the first equation from (22c) and the first variational problem from (34d), thus obtaining

$$
\int_{\Gamma_{1,2}^{o}}\left(\nabla w_{1,2} \cdot \nabla f_{\mu_{1,2 ; h}}+w_{1,2} f_{\mu_{1,2 ; h}}\right) d \Omega=\tilde{\mathbf{U}}_{1,2}^{T} \int_{\Gamma_{1,2}} \mathbf{H}_{1,2} \gamma_{\Gamma_{1,2}}\left(w_{1,2}\right) d \Gamma,
$$

for any $w_{1,2} \in H^{1}\left(\Gamma_{1,2}^{o}\right)$. In order to find an approximation $\tilde{f}_{\mu_{1,2 ; h}}$ for $f_{\mu_{1,2 ; h}}$ within the meshfree space $\mathcal{M}\left(\Gamma_{1,2}^{o}\right)$, we represent it as $\tilde{f}_{\mu_{1,2 ; h}}=\mathbf{E}_{1,2}^{T} \tilde{\mathbf{F}}_{1,2}$, as in (36a), where the (unknown) vector of coefficients $\tilde{\mathbf{F}}_{1,2}$

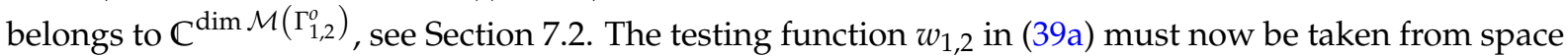
$\mathcal{M}\left(\Gamma_{1,2}^{o}\right)$, and so we denote it by $w_{1,2 ; h}$. It is represented likewise as $w_{1,2 ; h}=\mathbf{E}_{1,2}^{T} \tilde{\mathbf{W}}_{1,2}$, and its trace is represented as $\gamma_{\Gamma_{1,2}}\left(w_{1,2 ; h}\right)=\mathbf{R}_{1,2}^{T} \tilde{\mathbf{W}}_{1,2}$, in accordance with (36a) and (36b). After substituting these expansions, the discrete version of problem (39a) becomes

$$
\tilde{\mathbf{W}}_{1,2}^{T} \overline{\mathbf{Q}}_{1,2} \tilde{\mathbf{F}}_{1,2}=\tilde{\mathbf{W}}_{1,2}^{T} \overline{\mathbf{N}}_{1,2} \tilde{\mathbf{U}}_{1,2}
$$

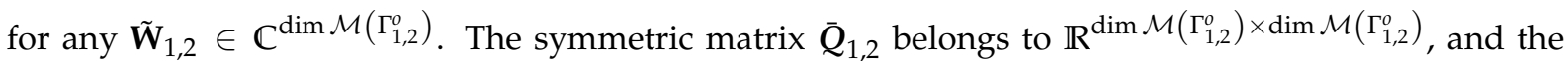

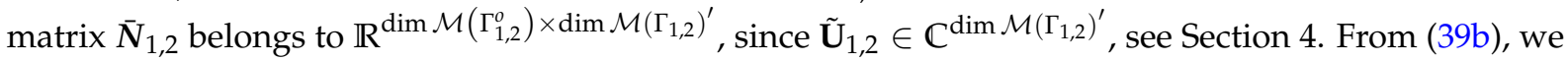
obtain $\bar{Q}_{1,2} \tilde{\mathbf{F}}_{1,2}=\overline{\mathbf{N}}_{1,2} \tilde{\mathbf{U}}_{1,2}$, and the coefficient vector $\tilde{\mathbf{F}}_{1,2}$ can be retrieved as

$$
\tilde{\mathbf{F}}_{1,2}=\bar{Q}_{1,2}^{-1} \bar{N}_{1,2} \tilde{\mathbf{U}}_{1,2}
$$

The same procedure above can be applied to find the approximations $\tilde{f}_{\mu_{2,4 ; h}}$ and $\tilde{f}_{\mu_{3,4 ; h}}$. These are expanded as $\tilde{f}_{\mu_{2,4 ; h}}=\mathbf{E}_{2,4}^{T} \tilde{\mathbf{F}}_{2,4}$ and $\tilde{f}_{\mu_{3,4 ; h}}=\mathbf{E}_{3,4}^{T} \tilde{\mathbf{F}}_{3,4}$, after (36a), and the unknown coefficients are given by

$$
\begin{aligned}
\tilde{\mathbf{F}}_{2,4} & =\bar{Q}_{2,4}^{-1} \bar{N}_{2,4} \tilde{\mathbf{U}}_{2,4} \\
\tilde{\mathbf{F}}_{3,4} & =\bar{Q}_{3,4}^{-1} \bar{N}_{3,4} \tilde{\mathbf{U}}_{3,4} .
\end{aligned}
$$


Using (34b), the norm $\left\|\mathbf{f}_{\boldsymbol{\mu}_{h}}\right\|_{\mathcal{Z}}$ in the denominator of inf-sup condition (38) can be approximated as

$$
\begin{aligned}
\left\|\mathbf{f}_{\boldsymbol{\mu}_{h}}\right\|_{\mathcal{Z}}^{2}=\left\|\left(f_{\mu_{1,2 ; h}}, f_{\mu_{2,4 ;}, f} f_{\mu_{3,4 ;}}\right)\right\|_{\mathcal{Z}}^{2} & \stackrel{\text { def }}{=}\left\|f_{\mu_{1,2 ; h}}\right\|_{H^{1}\left(\Gamma_{1,2}^{o}\right)}^{2}+\left\|f_{\mu_{2,4 ; h}}\right\|_{H^{1}\left(\Gamma_{2,4}^{o}\right)}^{2}+\left\|f_{\mu_{3,4 ; h}}\right\|_{H^{1}\left(\Gamma_{3,4}^{o}\right)}^{2} \\
& \cong\left\|\tilde{f}_{\mu_{1,2, h}}\right\|_{H^{1}\left(\Gamma_{1,2}^{o}\right)}^{2}+\left\|\tilde{f}_{\mu_{2,4 ; h}}\right\|_{H^{1}\left(\Gamma_{2,4}^{o}\right)}^{2}+\left\|\tilde{f}_{\mu_{3,4 ; h}}\right\|_{H^{1}\left(\Gamma_{3,4}^{o}\right)}^{2} .
\end{aligned}
$$

The first term of the sum above is expanded as

$$
\begin{aligned}
\left\|\tilde{f}_{\mu_{1,2 ; h}}\right\|_{H^{1}\left(\Gamma_{1,2}^{o}\right)}^{2} \stackrel{\text { def }}{=} \int_{\Gamma_{1,2}^{o}}\left(\nabla \tilde{f}_{\mu_{1,2 ; h}} \cdot \nabla \tilde{f}_{\mu_{1,2 ; h}}^{*}+\tilde{f}_{\mu_{1,2 ; h}} \tilde{f}_{\mu_{1,2 ; h}}^{*}\right) d \Omega \\
=\tilde{\mathbf{F}}_{1,2}^{\dagger} \bar{Q}_{1,2} \tilde{\mathbf{F}}_{1,2} \\
=\tilde{\mathbf{U}}_{1,2}^{+} \overline{\mathbf{N}}_{1,2}^{+}\left(\overline{\mathbf{Q}}_{1,2}^{-1}\right)^{\dagger} \overline{\mathbf{Q}}_{1,2} \overline{\mathbf{Q}}_{1,2}^{-1} \overline{\mathbf{N}}_{1,2} \tilde{\mathbf{U}}_{1,2} \\
=\tilde{\mathbf{U}}_{1,2}^{+} \overline{\mathbf{N}}_{1,2}^{T}\left(\overline{\mathbf{Q}}_{1,2}^{-1}\right)^{T} \overline{\mathbf{N}}_{1,2} \tilde{\mathbf{U}}_{1,2} \\
=\tilde{\mathbf{U}}_{1,2}^{+} \overline{\mathbf{N}}_{1,2}^{T} \overline{\mathbf{Q}}_{1,2}^{-1} \overline{\mathbf{N}}_{1,2} \tilde{\mathbf{U}}_{1,2},
\end{aligned}
$$

where we used the discretized representation $\tilde{f}_{\mu_{1,2 ; h}}=\mathbf{E}_{1,2}^{T} \tilde{\mathbf{F}}_{1,2}$ (see (36a)). The matrix $\bar{Q}_{1,2}$ is the same as that which appears in (39b). The vector $\tilde{\mathbf{F}}_{1,2}$ is given by (39c). The matrices $\bar{Q}_{1,2}$ and $\bar{N}_{1,2}$ are realvalued, which implies that $\overline{\boldsymbol{N}}_{1,2}^{\dagger}=\overline{\boldsymbol{N}}_{1,2}^{T}$ and $\left(\overline{\mathbf{Q}}_{1,2}^{-1}\right)^{\dagger}=\left(\overline{\boldsymbol{Q}}_{1,2}^{-1}\right)^{T}$. Finally, since $\overline{\mathbf{Q}}_{1,2}$ is symmetric, $\left(\bar{Q}_{1,2}^{-1}\right)^{T}=\bar{Q}_{1,2}^{-1}$. Applying the same reasoning to the second and third terms of (40a), we obtain

$$
\begin{aligned}
& \left\|\tilde{f}_{\mu_{2,4 ; h}}\right\|_{H^{1}\left(\Gamma_{2,4}^{o}\right)}^{2}=\tilde{\mathbf{U}}_{2,4}^{\dagger} \bar{N}_{2,4}^{T} \bar{Q}_{2,4}^{-1} \bar{N}_{2,4} \tilde{\mathbf{U}}_{2,4}, \\
& \left\|\tilde{f}_{\mu_{3,4 ; h}}\right\|_{H^{1}\left(\Gamma_{3,4}^{o}\right)}^{2}=\tilde{\mathbf{U}}_{3,4}^{\dagger} \bar{N}_{3,4}^{T} \bar{Q}_{3,4}^{-1} \bar{N}_{3,4} \tilde{\mathbf{U}}_{3,4} .
\end{aligned}
$$

Using (40b)-(40d), the sum (40a) can be rewritten in matrix form as

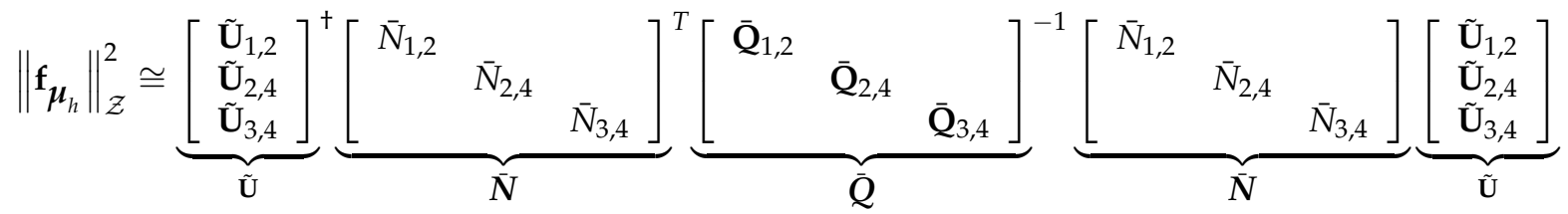

$$
\begin{aligned}
& =\tilde{\mathbf{U}}^{\dagger} \overline{\boldsymbol{N}}^{T} \overline{\mathbf{Q}}^{-1} \overline{\mathbf{N}} \tilde{\mathbf{U}}
\end{aligned}
$$

where the symmetric matrix $\bar{Q}$ belongs to $\mathbb{R}^{\operatorname{dim} \mathcal{Z}_{h} \times \operatorname{dim} \mathcal{Z}_{h}}$ and the matrix $\bar{N}$ belongs to $\mathbb{R}^{\operatorname{dim} \mathcal{Z}_{h} \times \operatorname{dim} \mathcal{Y}_{h}}$. Finally, the inf-sup condition (38) becomes equivalent to:

There is a constant $\beta_{h}>0$ such that

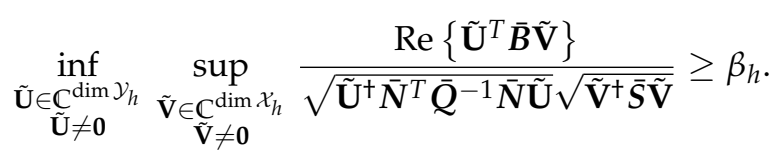

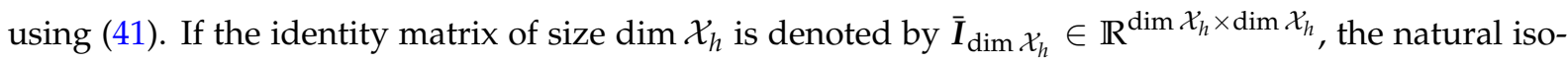
morphism between $\mathbb{R}^{2 \operatorname{dim} \mathcal{X}_{h}}$ and $\mathbb{C}^{\operatorname{dim} \mathcal{X}_{h}}$ can be represented by the matrix $\overline{\mathbf{J}}_{\operatorname{dim}} \mathcal{X}_{h} \stackrel{\operatorname{def}}{=}\left[\overline{\boldsymbol{I}}_{\operatorname{dim}} \mathcal{X}_{h}, j \overline{\boldsymbol{I}}_{\operatorname{dim}} \mathcal{X}_{h}\right] \in$ $\mathbb{C}^{\operatorname{dim} \mathcal{X}_{h} \times 2 \operatorname{dim} \mathcal{X}_{h}}$, via the map $\tilde{\mathfrak{v}} \longmapsto \overline{\mathbf{J}}_{\operatorname{dim} \mathcal{X}_{h}} \tilde{\mathfrak{v}}$. Analogously, the natural isomorphism between $\mathbb{R}^{2 \operatorname{dim} \mathcal{Y}_{h}}$

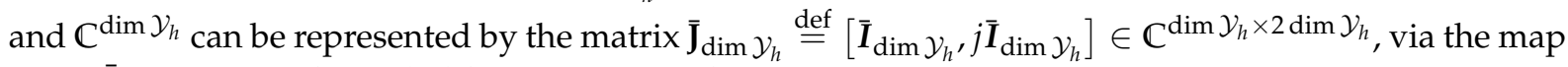
$\tilde{\mathfrak{u}} \longmapsto \overline{\mathbf{J}}_{\operatorname{dim}} \mathcal{Y}_{h} \tilde{\mathfrak{u}}$. Condition (42) becomes equivalent to: 
There is a constant $\beta_{h}>0$ such that

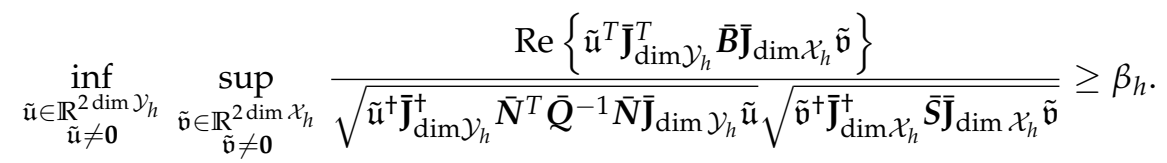

The vectors $\tilde{\mathfrak{u}}$ and $\tilde{\mathfrak{v}}$ are real-valued, which implies that $\tilde{\mathfrak{u}}^{\dagger}=\tilde{\mathfrak{u}}^{T}$ and $\tilde{\mathfrak{v}}^{\dagger}=\tilde{\mathfrak{v}}^{T}$. The numerator in (43)

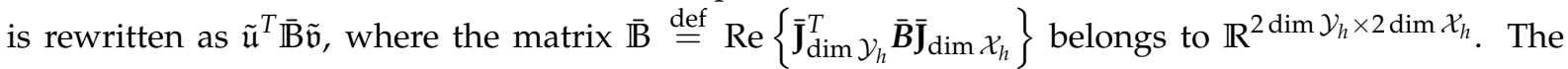
term inside the first square root in the denominator of (43) is rewritten as $\tilde{\mathfrak{u}}^{T} \overline{\mathbf{M}} \tilde{\mathfrak{u}}$, where the matrix

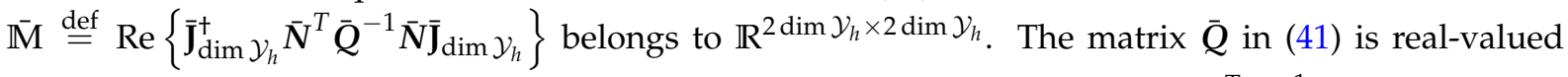
and symmetric, and it can be verified that the imaginary part of matrix $\overline{\mathbf{J}}_{\operatorname{dim}}^{+} \mathcal{Y}_{h} \bar{N}^{T} \bar{Q}^{-1} \bar{N} \overline{\mathbf{J}}_{\operatorname{dim} \mathcal{Y}_{h}}$ satisfies $\tilde{\mathfrak{u}}^{\dagger} \operatorname{Im}\left\{\overline{\mathbf{J}}_{\operatorname{dim} \mathcal{Y}_{h}}^{\dagger} \bar{N}^{T} \bar{Q}^{-1} \bar{N} \overline{\mathbf{J}}_{\operatorname{dim} \mathcal{Y}_{h}}\right\} \tilde{\mathfrak{u}}=0$. The term inside the second square root in the denominator of

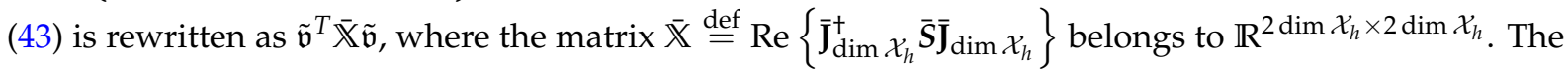
matrix $\bar{S}$ in (30b) is real-valued and symmetric, and it can be verified that the imaginary part of matrix $\overline{\mathbf{J}}_{\operatorname{dim} \mathcal{X}_{h}}^{\dagger} \bar{S} \overline{\mathbf{J}}_{\operatorname{dim} \mathcal{X}_{h}}$ satisfies $\tilde{\mathfrak{v}}^{\dagger} \operatorname{Im}\left\{\overline{\mathbf{J}}_{\operatorname{dim} \mathcal{X}_{h}}^{\dagger} \bar{S} \overline{\mathbf{J}}_{\operatorname{dim} \mathcal{X}_{h}}\right\} \tilde{\mathfrak{v}}=0$. The inf-sup condition (43) assumes its final form, becoming equivalent to:

There is a constant $\beta_{h}>0$ such that

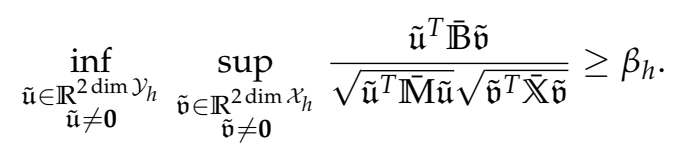

Analogously to (31), condition (44) is written entirely in terms of real-valued vectors and matrices.

\section{DEMONSTRATION OF THE MFS PROCEDURE}

In this section we illustrate the application of the MFS procedures from Section 4 to a particular scattering problem. The computational details and further solutions are given in the companion paper, see reference [10]. The problem refers to the scattering of a plane wave by a circular object. In this setting, we have a single object, corresponding to a circle, see Fig. 5(a). This geometric arrangement agrees with the assumptions made in Section 2.1. The material properties in regions $\Omega_{1}$ and $\Omega_{2}$ are $\rho_{1}=8.94 \times 10^{3} \mathrm{~kg} / \mathrm{m}^{3}$ and $\rho_{2}=1 \times 10^{3} \mathrm{~kg} / \mathrm{m}^{3}$ (density), and $K_{1}=123 \times 10^{9} \mathrm{~Pa}$ and $K_{2}=2.28 \times 10^{9} \mathrm{~Pa}$ (bulk modulus). The 'relative' values are $\rho_{1, \text { rel }}=8.94$ and $\rho_{2, \text { rel }}=1$, and $K_{1, \text { rel }}=53.95$ and $K_{2, \text { rel }}=1$, according to (4a) and (4b). We use the reference frequency of $f_{0}=100 \mathrm{kHz}$.

The reference wavenumber $k_{0}$ associated with the host medium $\Omega_{2}$ is $k_{0}=\omega_{0} / c=2 \pi f_{0} \sqrt{\rho_{2} / K_{2}}=$ $416.11 \mathrm{rad} / \mathrm{m}$. Consequently, the reference wavelength of a (hypothetical) plane wave with frequency $f_{0}$ propagating in $\Omega_{2}$ is given by $\lambda_{0}=2 \pi / k_{0}=15.10 \times 10^{-3} \mathrm{~m}$. Region $\Omega_{1}$ is a circle with radius equal to $\lambda_{0}$, and the outer circular boundary $\Gamma_{R}$ has its radius given by $R=2.15 \lambda_{0}$. Region $\Omega_{1}$ is simply connected, and region $\Omega_{2}$ (the host medium) is not simply-connected, as expected (see Section 2.1). This problem has a single interface, indicated by $\Gamma_{1,2}$ in Fig. 5(a).

We consider a unit plane wave with frequency $f$ equal to the reference frequency, i.e., $f=f_{0}$, with the wavenumber $k=k_{0}$. This wave propagates along the unit vector $\hat{k}=[1,0]$ (i.e., from left to right), and the incident field is therefore given by $p^{i n c}(x)=e^{-j k \hat{k} \cdot x}=e^{-j k x} \mathrm{~N} / \mathrm{m}^{2}, x \in \bar{\Omega}_{2}$.

In the discretization process, we set up three finite sphere systems, as illustrated in Figs. 5(b)-5(d). The closure of region $\Omega_{1}$ is covered with 549 balls, the closure of region $\Omega_{2}$ is covered with 1996 balls, and the interface $\Gamma_{1,2}$ is covered with 81 balls. The real and imaginary parts of the predicted total pressure field $\boldsymbol{p}_{h}=\left(p_{1 ; h}, p_{2 ; h}\right)$, as well as its modulus, are shown in Figs. 6(a)-6(c). We apply a close zoom to the solutions calculated along the line segment $0.8 \lambda_{0} \leq x \leq 1.2 \lambda_{0}, y=0$, shown in Figs. $6 \mathrm{a}$ and $6 \mathrm{~b}$. This line segment crosses the interface, and we compare the meshfree solutions thus obtained with FEM solutions (using quadratic Lagrange triangular elements) calculated along the same segment. The results for both real and imaginary parts are shown in Figs. $7(\mathrm{a})$ and $7(\mathrm{~b})$, respectively. The solutions provided by both 


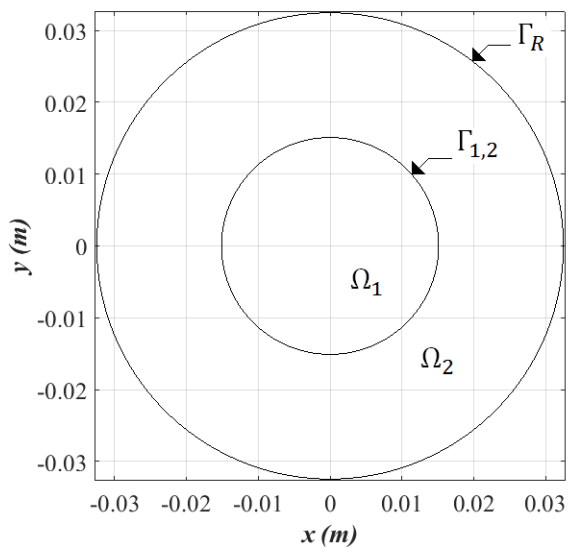

(a)

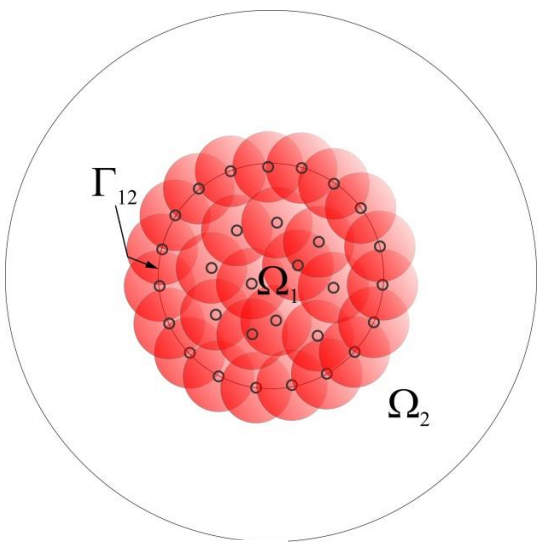

(c)

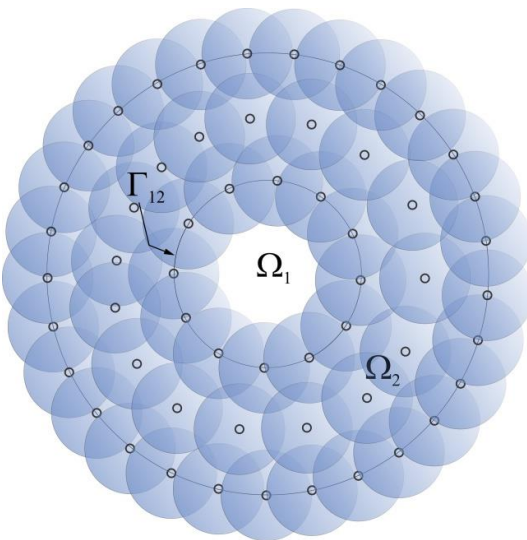

(b)

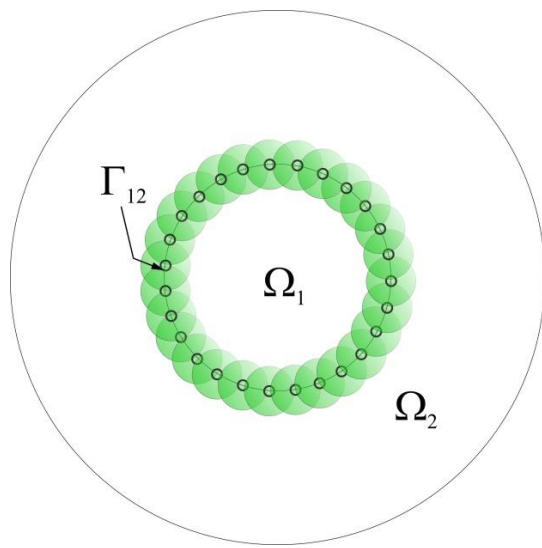

(d)

Fig. 5. Scattering by a circular object. (a) Geometry of the problem. (b) Balls in a finite sphere system over region $\Omega_{2}$ and along its boundary $\partial \Omega_{2}=\Gamma_{1,2} \cup \Gamma_{R}$. (c) Balls in a finite sphere system covering $\Omega_{1}$ and $\partial \Omega_{1}=\Gamma_{1,2}$. (d) Balls in a finite sphere system covering the interface $\Gamma_{1,2}$

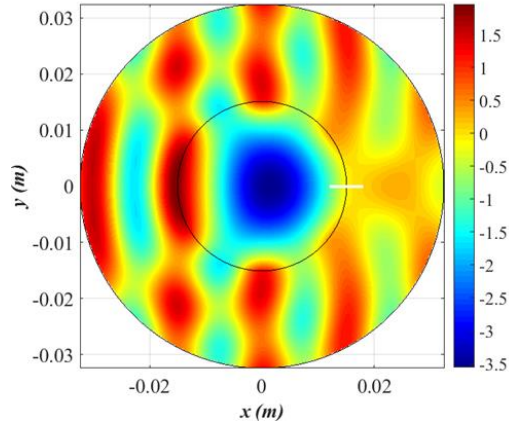

(a)

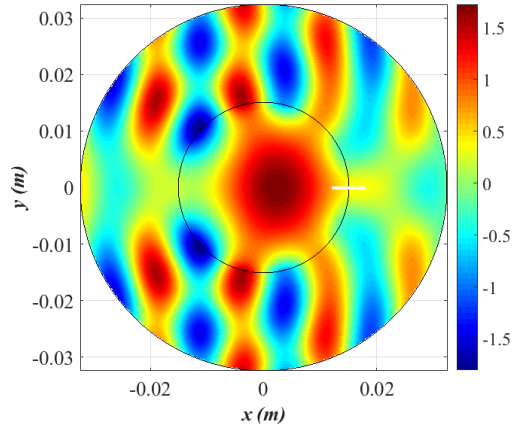

(b)

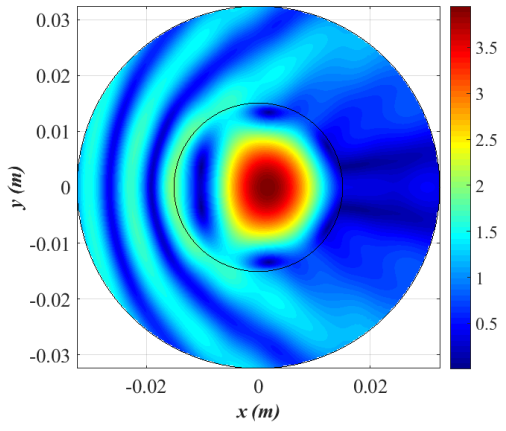

(c)

Fig. 6. Scattering by a circular object. (a) Real part of the meshfree solution throughout the computational domain. (b) Imaginary part of the meshfree solution. (c) The modulus of the meshfree solution

methods agree with each other, and the MFS solution reproduced the discontinuity in the gradients accurately. No oscillations are observed, according to our objective in the MFS formulation. 


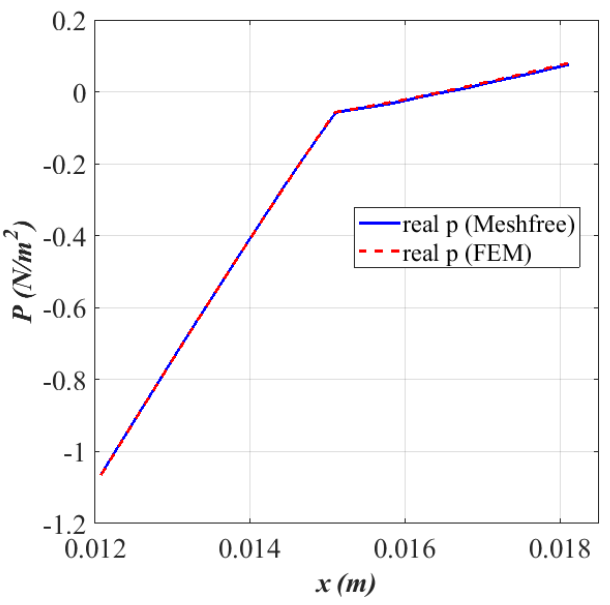

(a)

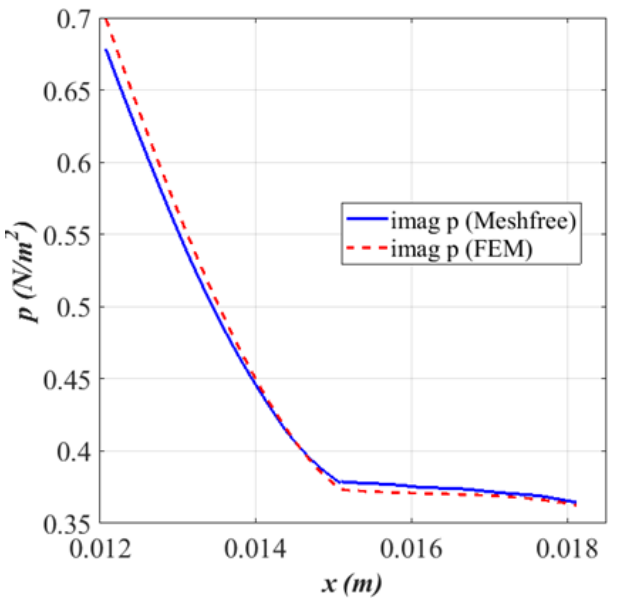

(b)

Fig. 7. (a) Real part of meshfree and FEM solutions along the segment $0.8 \lambda_{0} \leq x \leq 1.2 \lambda_{0}, y=0$ (shown in white in Fig. 6(a)). (b) Imaginary part of meshfree and FEM solutions along the same segment (shown in white in Fig. 6(b))

\section{DEMONSTRATION OF THE INF-SUP TESTING}

For a given discretization characterized by length $h$, Sections 5-7 showed that the discrete problem (23) is well-posed (its solution exists, is unique, and depends continuously on the data) if the inf-sup conditions (31) and (44) are satisfied. In order to verify if these conditions hold, we observe that

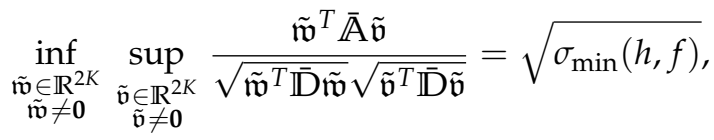

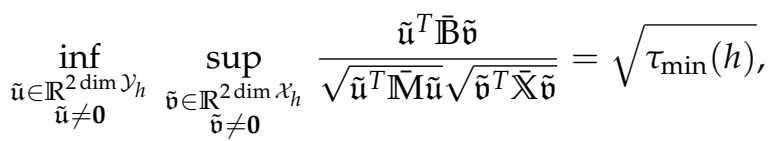

where $\sigma_{\min }(h, f)$ and $\tau_{\min }(h)$ are the smallest eigenvalues in the problems

$$
\begin{gathered}
\overline{\mathbb{A}} \overline{\mathbb{D}}^{-1} \overline{\mathbb{A}}^{T} \tilde{\mathfrak{w}}_{i}=\sigma_{i} \overline{\mathbb{D}} \tilde{\mathfrak{w}}_{i}, \\
\overline{\mathbb{B}} \overline{\mathbb{X}}{ }^{-1} \overline{\mathbb{B}}^{T} \tilde{\mathfrak{u}}_{i}=\tau_{i} \overline{\mathbb{M}} \tilde{\mathfrak{u}}_{i},
\end{gathered}
$$

see [6]. These smallest eigenvalues depend on the discretization length $h$. The matrix $\overline{\mathbb{A}}$ in (31) is given in terms of the matrix $\bar{A}$ in (24a), which is the discrete representation of the bilinear form $a$ in (19a). This bilinear form involves the wavenumber $k$, which is calculated as $k=\omega / c$ (see Sections 2 and 8 ). Since $\omega=2 \pi f$, it follows that the matrix $\overline{\mathbb{A}}$ depends on the frequency $f$ of the incident field. The smallest eigenvalue $\sigma_{\min }$ in (45a) therefore depends on both $h$ and $f$.

The idea behind the inf-sup test is to evaluate the behavior of these smallest eigenvalues as the discretization is refined, i.e., as $h$ becomes successively smaller [28]. If these eigenvalues stabilize at a value larger than zero, the inf-sup test is passed for the discretization scheme used. If in this way both inf-sup tests are passed, we can conclude that the discrete problems retain their well-posedness and hence the MFS formulation results in stable solutions for any $h$.

We demonstrate now the stability of the scattering problem considered in Section 8. Further discussion and more examples are given in [10]. We consider a sequence of 15 pairs of meshfree spaces $\mathcal{X}_{h}$ and $\mathcal{Y}_{h}$, from the coarsest to the finest. For each pair, the discretization length $h$ is established as follows: for each ball in the discretization, we calculate the distance between its center and that of its closest neighboring ball; then $h$ is defined as the largest of these distances. The number of balls over $\Omega_{1}$ varies from 57 to 401 , the number of balls over $\Omega_{2}$ varies from 227 to 1473, and the number of balls over $\Gamma_{1,2}$ varies from 25 to 69 . Since region $\Omega_{1}$ is simply connected (see Fig. 5(a)), it follows that the region 
interior to curve $\Gamma_{1,2}$ is the region $\Omega_{1}$, i.e., $\Gamma_{1,2}^{o}=\Omega_{1}$. We thus reuse the finite sphere systems over $\Omega_{1}$ as the finite sphere systems over $\Gamma_{1,2}^{o}$ (see Section 7.2).

In Section 8, the incident plane wave has a frequency of $f=f_{0}=100 \mathrm{kHz}$, so that the wavenumber of the problem is $k=k_{0}=416.11 \mathrm{rad} / \mathrm{m}$. For the first inf-sup condition, we first consider a frequency $f=50 \mathrm{kHz}$, so that the wavenumber of the problem is $k=\omega / c=2 \pi f \sqrt{\rho_{2} / K_{2}}=208.06 \mathrm{rad} / \mathrm{m}$, and calculate the smallest eigenvalues $\sigma_{\min }(h, 50 \mathrm{kHz})$ in (45a) for the sequence of 15 discretizations. Next, we consider the new frequency $f=60 \mathrm{kHz}$, determine the new wavenumber $k$, and proceed to calculate the smallest eigenvalues $\sigma_{\min }(h, 60 \mathrm{kHz})$ for the sequence of 15 discretizations. This process is repeated until $f=300 \mathrm{kHz}$ (i.e., we consider increments of $10 \mathrm{kHz}$ in the frequency). Using these results, we plot the graph corresponding to the values $\sqrt{\sigma_{\min }(h, f)}$ in Fig. 8(a). We notice that for each frequency $f$, the values $\sqrt{\sigma_{\min }(h, f)}$ are positive and stabilize around a positive value (i.e., they neither increase nor decrease with $h$ ). This implies that for each fixed frequency $f$, the test for the first inf-sup condition (31) has been passed. At any given $f$ there is a negligible variation with $h$ in Fig. 8(a), hence the figure shows a profile with parallel lines along the $h$ axis. The inf-sup values may appear to be small but what really matters is that they do not decrease with $h$, i.e., they stabilize as the discretization becomes finer, see also $[46,47]$. Hence we see that the inf-sup test is passed for all frequencies considered. Fig. 8(b) shows the values $\sqrt{\tau_{\min }(h)}$ for the second inf-sup condition. We observe that they are all positive and stabilize at a positive value, so that the test for the second inf-sup condition (44) is also passed. We conclude that for this example and this range of frequencies (which are reasonable to consider in finite element analysis) the proposed MFS procedure is stable and yields reliable solutions.

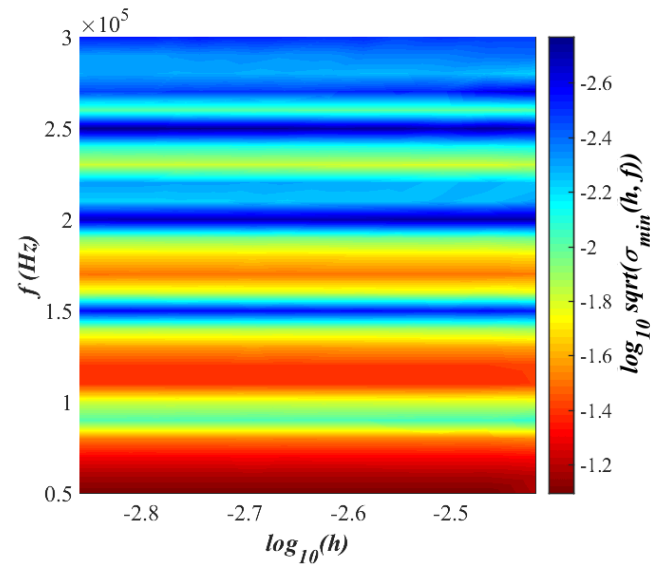

(a)

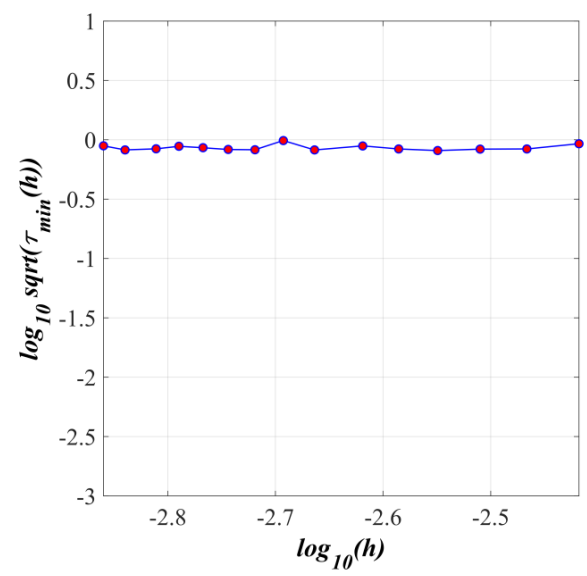

(b)

Fig. 8. The inf-sup values, scattering by a circular object (Section 8). (a) First inf-sup condition, $\log _{10} \sqrt{\sigma_{\min }(h, f)}$ as a function of $\log _{10} h$ and $f$. (b) Second inf-sup condition, $\log _{10} \sqrt{\tau_{\min }(h)}$ as a function of $\log _{10} h$

\section{CONCLUDING REMARKS}

We provided a derivation of the method of finite spheres equations for solutions of acoustic pressure fields in nonhomogeneous media. Lagrange multiplier fields arise naturally leading to a mixed formulation. Using the solution scheme, the well-posedness of the discrete problems is governed by two inf-sup conditions. These conditions are difficult to work with, particularly the second one, due to the $H^{-1 / 2}$ dual norm. We presented an analysis able to cover the most general case when the bilinear forms may assume complex values. Our result is that we obtained stronger conditions, simpler to deal with and written entirely in terms of real-valued matrices and vectors. These conditions pave the 
way for use of the inf-sup test, an application of which is given in Section 9. The goal of this test is to numerically verify the stability of the mixed formulation.

The analysis presented uses the geometrical setting given in Fig. 1 as an example, which is general enough to treat the scattering of waves in simply connected regions (such as $\Omega_{3}$ ) and also in not simplyconnected regions (such as $\Omega_{2}$ ). Of course, the discussion can be adapted to any geometry, as long as the assumptions made still hold.

The ideas presented here can also be developed and built upon to analyze the inf-sup conditions of scattering problems posed in domains in which the interfaces between media of different material properties are open curves (see for example [10]). Furthermore, the development given also provides a basis for use with other meshfree methods and for use in analyses using the overlapping finite element schemes $[7,14]$.

\section{ACKNOWLEDGMENT}

This research work was supported by the Brazilian Ministry of Education (CAPES/PNPD), and by the National Council for Scientific and Technological Development (CNPq), under grant 150699/2019-0.

\section{REFERENCES}

[1] S. De and K. J. Bathe. The method of finite spheres. Computational Mechanics, 25, (4), (2000), pp. 329-345. https://doi.org/10.1007/s004660050481.

[2] J. J. Monaghan. An introduction to SPH. Computer Physics Communications, 48, (1988), pp. 89-96.

[3] T. Belytschko, Y. Y. Lu, and L. Gu. Element-free Galerkin methods. International journal for numerical methods in engineering, 37, (2), (1994), pp. 229-256. https://doi.org/10.1002/nme.1620370205.

[4] S. Atluri and S. Shen. The meshless local Petrov-Galerkin method: A simple and less-costly alternative to the finite-element and boundary element methods. CMES: Computer Modeling in Engineering \& Sciences, 3, (2002), pp. 11-51.

[5] G. R. Liu. Meshfree methods: moving beyond the finite element method. 2nd edition, CRC Press, (2010).

[6] K. J. Bathe. Finite element procedures. Prentice Hall, (1996). 2nd edition, K. J. Bathe, Watertown MA, (2014). Higher Education Press China, (2016).

[7] K. J. Bathe and L. Zhang. The finite element method with overlapping elements-a new paradigm for CAD driven simulations. Computers \& Structures, 182, (2017), pp. 526-539. https://doi.org/10.1016/j.compstruc.2016.10.020.

[8] L. Zhang, K. T. Kim, and K. J. Bathe. The new paradigm of finite element solutions with overlapping elements in CAD-Computational efficiency of the procedure. Computers \& Structures, 199, (2018), pp. 1-17. https://doi.org/10.1016/j.compstruc.2018.01.003.

[9] W. L. Nicomedes, K. J. Bathe, F. J. S. Moreira, and R. C. Mesquita. Meshfree analysis of electromagnetic wave scattering from conducting targets: Formulation and computations. Computers \& Structures, 184, (2017), pp. 3652. https://doi.org/10.1016/j.compstruc.2017.01.014.

[10] W. L. Nicomedes, K. J. Bathe, F. J. S. Moreira, and R. C. Mesquita. Mesquita, Acoustic scattering in nonhomogeneous media and the problem of discontinuous gradients: Analysis and inf-sup stability in the method of finite spheres. submitted, (2020).

[11] F. B. Jensen, W. A. Kuperman, M. B. Porter, and H. Schmidt. Computational ocean acoustics. 2nd edition, Springer, (2011).

[12] F. Brezzi and M. Fortin. Mixed and hybrid finite element methods. Springer, (1991).

[13] D. Boffi, F. Brezzi, and M. Fortin. Mixed finite element methods and applications. Springer, (2013).

[14] Y. Chai and K. J. Bathe. Transient wave propagation in inhomogeneous media with enriched overlapping triangular elements. Computers \& Structures, 237, (2020). https://doi.org/10.1016/j.compstruc.2020.106273.

[15] L. Tartar. An introduction to Sobolev spaces and interpolation spaces. Springer, (2007).

[16] G. Leoni. A first course in Sobolev spaces. 2nd edition, American Mathematical Society, (2017).

[17] E. DiBenedetto. Real analysis. 2nd edition, Birkhäuser, (2016).

[18] C. Farhat, I. Harari, and U. Hetmaniuk. A discontinuous Galerkin method with Lagrange multipliers for the solution of Helmholtz problems in the mid-frequency regime. Computer Methods in Applied Mechanics and Engineering, 192, (11-12), (2003), pp. 1389-1419. https://doi.org/10.1016/s0045-7825(02)00646-1.

[19] I. Kalashnikova, C. Farhat, and R. Tezaur. A discontinuous enrichment method for the finite element solution of high Péclet advection-diffusion problems. Finite elements in Analysis and Design, 45, (4), (2009), pp. 238-250. https://doi.org/10.1016/j.finel.2008.10.009. 
[20] E. Grosu and I. Harari. Three-dimensional element configurations for the discontinuous enrichment method for acoustics. International Journal for Numerical Methods in Engineering, 78, (11), (2009), pp. 1261-1291. https://doi.org/10.1002/nme.2525.

[21] C. Farhat, I. Kalashnikova, and R. Tezaur. A higher-order discontinuous enrichment method for the solution of high péclet advection-diffusion problems on unstructured meshes. International Journal for Numerical Methods in Engineering, 81, (5), (2010), pp. 604-636. https://doi.org/10.1002/nme.2706.

[22] I. Kalashnikova, R. Tezaur, and C. Farhat. A discontinuous enrichment method for variable-coefficient advection-diffusion at high Péclet number. International Journal for Numerical Methods in Engineering, 87, (1-5), (2010), pp. 309-335. https://doi.org/10.1002/nme.3058.

[23] F. Brezzi and K. J. Bathe. A discourse on the stability conditions for mixed finite element formulations. Computer Methods in Applied Mechanics and Engineering, 82, (1-3), (1990), pp. 27-57. https://doi.org/10.1016/00457825(90)90157-h.

[24] N. El-Abbasi and K. J. Bathe. Stability and patch test performance of contact discretizations and a new solution algorithm. Computers \& Structures, 79, (16), (2001), pp. 1473-1486. https://doi.org/10.1016/s00457949(01)00048-7.

[25] N. Moës, E. Béchet, and M. Tourbier. Imposing Dirichlet boundary conditions in the extended finite element method. International Journal for Numerical Methods in Engineering, 67, (12), (2006), pp. 1641-1669. https://doi.org/10.1002/nme.1675.

[26] É. Béchet, N. Moës, and B. Wohlmuth. A stable Lagrange multiplier space for stiff interface conditions within the extended finite element method. International Journal for Numerical Methods in Engineering, 78, (8), (2009), pp. 931-954. https://doi.org/10.1002/nme.2515.

[27] G. Ferté, P. Massin, and N. Moës. Interface problems with quadratic X-FEM: design of a stable multiplier space and error analysis. International Journal for Numerical Methods in Engineering, 100, (11), (2014), pp. 834-870. https://doi.org/10.1002/nme.4787.

[28] K. J. Bathe. The inf-sup condition and its evaluation for mixed finite element methods. Computers $\mathcal{E}$ Structures, 79, (2), (2001), pp. 243-252. https://doi.org/10.1016/s0045-7949(00)00123-1.

[29] D. Chapelle and K. J. Bathe. The inf-sup test. Computers \& Structures, 47, (4-5), (1993), pp. 537-545. https://doi.org/10.1016/0045-7949(93)90340-j.

[30] W. Bao, X. Wang, and K. J. Bathe. On the inf-sup condition of mixed finite element formulations for acoustic fluids. Mathematical Models and Methods in Applied Sciences, 11, (05), (2001), pp. 883-901. https://doi.org/10.1142/s0218202501001161.

[31] A. F. Peterson, S. L. Ray, R. Mittra, I. of Electrical, and E. Engineers. Computational methods for electromagnetics. IEEE Press Series on Electromagnetic Waves, (1998).

[32] L. C. Evans. Partial differential equations. 2nd edition, American Mathematical Society, (2010).

[33] J. T. Oden and J. N. Reddy. An introduction to the mathematical theory of finite elements. Dover, (2011).

[34] S. Salsa. Partial differential equations in action: from modelling to theory. 3rd edition, Springer, (2016).

[35] W. McLean. Strongly elliptic systems and boundary integral equations. Cambridge University Press, (2000).

[36] J. C. Nédélec. Acoustic and electromagnetic equations: integral representations for harmonic problems. Springer, (2001).

[37] D. Colton and R. Kress. Inverse acoustic and electromagnetic scattering theory. 3rd edition, Springer, (2013).

[38] V. Girault and P. A. Raviart. Finite element methods for Navier-Stokes equations: theory and algorithms. Springer, (1986).

[39] A. Ern and J. L. Guermond. Theory and practice of finite elements. Springer, (2004).

[40] F. Boyer and P. Fabrie. Mathematical tools for the study of the incompressible Navier-Stokes equations and related models. Springer, (2012).

[41] D. Chapelle and K. J. Bathe. The finite element analysis of shells-fundamentals. 2nd edition, Springer, (2011).

[42] P. G. Ciarlet. Linear and nonlinear functional analysis with applications. Society for Industrial and Applied Mathematics - SIAM, (2013).

[43] D. Chapelle and K. J. Bathe. On the ellipticity condition for model-parameter dependent mixed formulations. Computers \& Structures, 88, (9-10), (2010), pp. 581-587.

[44] F. Ihlenburg. Finite element analysis of acoustic scattering. Springer, (1988).

[45] A. Moiola and E. A. Spence. Is the Helmholtz equation really sign-indefinite? SIAM Review, 56, (2), (2014), pp. 274-312. https://doi.org/10.1137/120901301.

[46] K. J. Bathe, A. Iosilevich, and D. Chapelle. An inf-sup test for shell finite elements. Computers $\mathcal{E}$ Structures, 75, (5), (2000), pp. 439-456. https://doi.org/10.1016/s0045-7949(99)00213-8.

[47] Y. Ko and K. J. Bathe. Inf-sup testing of some three-dimensional low-order finite elements for the analysis of solids. Computers \& Structures, 209, (2018), pp. 1-13. https://doi.org/10.1016/j.compstruc.2018.07.006.

[48] P. Grisvard. Elliptic problems in nonsmooth domains. Society for Industrial and Applied Mathematics - SIAM, (2011). https://doi.org/10.1137/1.9781611972030.

[49] J. R. Munkres. Topology. 2nd edition, Pearson, (2000). 


\section{APPENDIX A. THE TRACE THEOREM}

The complete version of this theorem is discussed in detail in $[40,48]$.

Theorem A.1. Let $D$ be a bounded and open subset of $\mathbb{R}^{2}$ with a Lipschitz continuous boundary $\partial D$. The trace operator $\gamma_{\partial D}: H^{1}(D) \longrightarrow H^{1 / 2}(\partial D)$, which is linear and continuous, admits a right inverse, i.e., there is a linear and continuous operator $\gamma_{\partial D}^{-}: H^{1 / 2}(\partial D) \longrightarrow H^{1}(D)$ such that for any $g \in H^{1 / 2}(\partial D)$,

$$
\gamma_{\partial D}\left(\gamma_{\partial D}^{-}(g)\right)=g \text {, }
$$

and there is a positive constant $C_{D}>0$ such that for any $g \in H^{1 / 2}(\partial D)$,

$$
\left\|\gamma_{\partial D}^{-}(g)\right\|_{H^{1}(D)} \leq C_{D}\|g\|_{H^{1 / 2}(\partial D)} \text {. }
$$

\section{APPENDIX B. THE SPACE $H^{1 / 2}$}

Let $D$ be an open and bounded subset of $\mathbb{R}^{2}$ with Lipschitz continuous boundary $\partial D$. A function $t \in L^{2}(\partial D)$ belongs to the fractional Sobolev space $H^{1 / 2}(\partial D)$ if the Slobodeckij seminorm

$$
|t|_{H^{1 / 2}(\partial D)} \stackrel{\text { def }}{=}\left(\int_{\partial D} \int_{\partial D} \frac{|t(\boldsymbol{x})-t(\boldsymbol{y})|^{2}}{\|\boldsymbol{x}-\boldsymbol{y}\|^{2}} d \Gamma_{x} d \Gamma_{y}\right)^{\frac{1}{2}},
$$

is finite, where $d \Gamma_{x}$ and $d \Gamma_{y}$ denote the length measure on $\partial D$ parametrized by $x$ and $y$, respectively [15-17]. The norm in $H^{1 / 2}(\partial D)$ is given by

$$
\|t\|_{H^{1 / 2}(\partial D)} \stackrel{\text { def }}{=}\left(\|t\|_{L^{2}(\partial D)}^{2}+|t|_{H^{1 / 2}(\partial D)}^{2}\right)^{\frac{1}{2}} .
$$

Among the properties of the space $H^{1 / 2}$, we list:

1. The space $H^{1 / 2}(\partial D)$ is a Hilbert space, and it is the image of the trace operator $\gamma_{\partial D}$ introduced in Appendix A.

2. For any $t \in H^{1 / 2}(\partial D)$ and for any subset $\Gamma_{0} \subset \partial D$ (with nonzero measure), it follows from (B.1) that the restriction of $t$ to $\Gamma_{0}$ belongs to $H^{1 / 2}\left(\Gamma_{0}\right)$, i.e., $\left.t\right|_{\Gamma_{0}} \in H^{1 / 2}\left(\Gamma_{0}\right)$.

\section{APPENDIX C. THE THEOREM 3.2}

Proof (1). The linearity of $E_{\left[\sigma_{k}, \partial \Omega\right]}$ is obvious. We check for boundedness. Assume that $w \in H^{1 / 2}\left(\sigma_{k}\right)$ is arbitrary. It is clear that $E_{\left[\sigma_{k}, \partial \Omega\right]}(w) \in L^{2}(\partial \Omega)$, since

$$
\begin{aligned}
\left\|E_{\left[\sigma_{k}, \partial \Omega\right]}(w)\right\|_{L^{2}(\partial \Omega)}^{2} & \stackrel{\text { def }}{=} \int_{\partial \Omega}\left|E_{\left[\sigma_{k}, \partial \Omega\right]}(w)\right|^{2} d \Gamma \\
& =\sum_{i=1}^{c} \int_{\sigma_{i}}\left|E_{\left[\sigma_{k}, \partial \Omega\right]}(w)\right|^{2} d \Gamma=\int_{\sigma_{k}}|w|^{2} d \Gamma=\|w\|_{L^{2}\left(\sigma_{k}\right)}^{2}<\infty .
\end{aligned}
$$

The Slobodeckij seminorm of $E_{\left[\sigma_{k}, \partial \Omega\right]}(w)$ is:

$$
\begin{aligned}
\left|E_{\left[\sigma_{k}, \partial \Omega\right]}(w)\right|_{H^{1 / 2}(\partial \Omega)}^{2} \stackrel{\text { def }}{=} & \int_{\partial \Omega} \int_{\partial \Omega} \frac{\left|\left(E_{\left[\sigma_{k}, \partial \Omega\right]}(w)\right)(\boldsymbol{x})-\left(E_{\left[\sigma_{k}, \partial \Omega\right]}(w)\right)(\boldsymbol{y})\right|^{2}}{\|\boldsymbol{x}-\boldsymbol{y}\|^{2}} d \Gamma_{x} d \Gamma_{\boldsymbol{y}} \\
= & \sum_{i=1}^{c} \sum_{j=1}^{c} \int_{\sigma_{j}} \int_{\sigma_{i}} \frac{\left|\left(E_{\left[\sigma_{k}, \partial \Omega\right]}(w)\right)(\boldsymbol{x})-\left(E_{\left[\sigma_{k}, \partial \Omega\right]}(w)\right)(\boldsymbol{y})\right|^{2}}{\|\boldsymbol{x}-\boldsymbol{y}\|^{2}} d \Gamma_{x} d \Gamma_{\boldsymbol{y}} \\
= & \int_{\sigma_{k}} \int_{\sigma_{k}} \frac{|w(\boldsymbol{x})-w(\boldsymbol{y})|^{2}}{\|\boldsymbol{x}-\boldsymbol{y}\|^{2}} d \Gamma_{x} d \Gamma_{\boldsymbol{y}}+\sum_{\substack{j=1 \\
j \neq k}}^{c} \int_{\sigma_{j}} \int_{\sigma_{k}} \frac{|w(\boldsymbol{x})-0|^{2}}{\|\boldsymbol{x}-\boldsymbol{y}\|^{2}} d \Gamma_{x} d \Gamma_{\boldsymbol{y}} \\
& +\sum_{\substack{i=1 \\
i \neq k}}^{c} \int_{\sigma_{k}} \int_{\sigma_{i}} \frac{|0-w(\boldsymbol{y})|^{2}}{\|\boldsymbol{x}-\boldsymbol{y}\|^{2}} d \Gamma_{x} d \Gamma_{\boldsymbol{y}}+\sum_{\substack{i=1 \\
i \neq k}}^{c} \sum_{\substack{j=1 \\
j \neq k}}^{c} \int_{\sigma_{j}} \int_{\sigma_{i}} \frac{|0-0|^{2}}{\|\boldsymbol{x}-\boldsymbol{y}\|^{2}} d \Gamma_{x} d \Gamma_{\boldsymbol{y}},
\end{aligned}
$$


where we used the extension rule from (9a). The first term at the right side of the last equality in (C.2) is just $|w|_{H^{1 / 2}\left(\sigma_{k}\right)}^{2}$, according to (B.1). Since the connected components $\sigma_{1}, \ldots, \sigma_{\mathcal{C}}$ lie at a certain distance from each other, it means that for any $i, j=1, \ldots, c$, if $i \neq j$, then

$$
\|\boldsymbol{x}-\boldsymbol{y}\| \geq \operatorname{dist}\left(\sigma_{i}, \sigma_{j}\right)>0, \text { for all }(\boldsymbol{x}, \boldsymbol{y}) \in \sigma_{i} \times \sigma_{j} .
$$

Moreover, since $w$ is a function defined on $\sigma_{k},($ C.2) becomes

$$
\begin{aligned}
& \left|E_{\left[\sigma_{k}, \partial \Omega\right]}(w)\right|_{H^{1 / 2}(\partial \Omega)}^{2} \leq|w|_{H^{1 / 2}\left(\sigma_{k}\right)}^{2} \\
& +\sum_{\substack{j=1 \\
j \neq k}}^{c} \frac{1}{\operatorname{dist}\left(\sigma_{k}, \sigma_{j}\right)^{2}} \int_{\sigma_{j}}\left(\int_{\sigma_{k}}|w(\boldsymbol{x})|^{2} d \Gamma_{\boldsymbol{x}}\right) d \Gamma_{\boldsymbol{y}}+\sum_{\substack{i=1 \\
i \neq k}}^{c} \frac{1}{\operatorname{dist}\left(\sigma_{i}, \sigma_{k}\right)^{2}} \int_{\sigma_{k}}|w(\boldsymbol{y})|^{2}\left(\int_{\sigma_{i}} d \Gamma_{\boldsymbol{x}}\right) d \Gamma_{\boldsymbol{y}} .
\end{aligned}
$$

The term within the parentheses in the first integral above is $\|w\|_{L^{2}\left(\sigma_{k}\right)}^{2}$, whereas the term within parentheses in the second integral is just the length of $\sigma_{i}$, denoted by $\left|\sigma_{i}\right|$. Expression above becomes

$$
\left|E_{\left[\sigma_{k}, \partial \Omega\right]}(w)\right|_{H^{1 / 2}(\partial \Omega)}^{2} \leq|w|_{H^{1 / 2}\left(\sigma_{k}\right)}^{2}+\|w\|_{L^{2}\left(\sigma_{k}\right)}^{2} \underbrace{\left(\sum_{\substack{j=1 \\ j \neq k}}^{c} \frac{\left|\sigma_{j}\right|}{\operatorname{dist}\left(\sigma_{k}, \sigma_{j}\right)^{2}}+\sum_{\substack{i=1 \\ i \neq k}}^{c} \frac{\left|\sigma_{i}\right|}{\operatorname{dist}\left(\sigma_{i}, \sigma_{k}\right)^{2}}\right)}_{C} .
$$

Clearly, $C$ in (C.5) is a positive constant depending only on the geometry of the boundary $\partial \Omega$. It follows from (C.5) that the Slobodeckij seminorm of $E_{\left[\sigma_{k}, \partial \Omega\right]}(w)$ is finite, and thus $E_{\left[\sigma_{k}, \partial \Omega\right]}(w)$ belongs to $H^{1 / 2}(\partial \Omega)$ (see Appendix B). Moreover, according to (B.2),

$$
\begin{aligned}
\left\|E_{\left[\sigma_{k}, \partial \Omega\right]}(w)\right\|_{H^{1 / 2}(\partial \Omega)}^{2} & =\left\|E_{\left[\sigma_{k}, \partial \Omega\right]}(w)\right\|_{L^{2}(\partial \Omega)}^{2}+\left|E_{\left[\sigma_{k}, \partial \Omega\right]}(w)\right|_{H^{1 / 2}(\partial \Omega)}^{2} \\
& \leq\|w\|_{L^{2}\left(\sigma_{k}\right)}^{2}+C\|w\|_{L^{2}\left(\sigma_{k}\right)}^{2}+|w|_{H^{1 / 2}\left(\sigma_{k}\right)}^{2} \\
& \leq(1+C)\left(\|w\|_{L^{2}\left(\sigma_{k}\right)}^{2}+|w|_{H^{1 / 2}\left(\sigma_{k}\right)}^{2}\right) \\
& =(1+C)\|w\|_{H^{1 / 2}\left(\sigma_{k}\right)}^{2},
\end{aligned}
$$

where we used (C.1) and (C.5). Since the constant $1+C$ depends only on the geometry of $\partial \Omega$, it thus follows that $E_{\left[\sigma_{k}, \partial \Omega\right]}$ is a bounded operator from $H^{1 / 2}\left(\sigma_{k}\right)$ into $H^{1 / 2}(\partial \Omega)$.

Proof (2). Let $\mu \in H^{-1 / 2}(\partial \Omega)$ be arbitrary. We define the functional $\tilde{\mu}_{k}$ as

$$
\tilde{\mu}_{k}(w) \stackrel{\text { def }}{=}\left\langle\mu \mid E_{\left[\sigma_{k}, \partial \Omega\right]}(w)\right\rangle_{H^{1 / 2}(\partial \Omega)}
$$

for any $w \in H^{1 / 2}\left(\sigma_{k}\right)$. Linearity of $\tilde{\mu}_{k}$ is obvious. Boundedness follows from

$$
\begin{aligned}
\left|\tilde{\mu}_{k}(w)\right| & =\left|\left\langle\mu \mid E_{\left[\sigma_{k}, \partial \Omega\right]}(w)\right\rangle_{H^{1 / 2}(\partial \Omega)}\right| \\
& \leq\|\mu\|_{H^{-1 / 2}(\partial \Omega)}\left\|E_{\left[\sigma_{k}, \partial \Omega\right]}(w)\right\|_{H^{1 / 2}(\partial \Omega)} \\
& \leq \underbrace{\sqrt{1+C}\|\mu\|_{H^{-1 / 2}(\partial \Omega)}}_{D}\|w\|_{H^{1 / 2}\left(\sigma_{k}\right)^{\prime}}
\end{aligned}
$$

due to (C.6). The positive constant $D$ is independent of any particular choice of $w$. Since the functional $\tilde{\mu}_{k}$ is linear and bounded, it belongs to the dual space $H^{-1 / 2}\left(\sigma_{k}\right)$. We can therefore represent the action of $\tilde{\mu}_{k}$ on elements of $H^{1 / 2}\left(\sigma_{k}\right)$ as $\left\langle\tilde{\mu}_{k} \mid w\right\rangle_{H^{1 / 2}\left(\sigma_{k}\right)} \stackrel{\text { def }}{=} \tilde{\mu}_{k}(w)$, for any $w \in H^{1 / 2}\left(\sigma_{k}\right)$. So for each $\mu \in$ $H^{-1 / 2}(\partial \Omega)$ there exists a corresponding $\tilde{\mu}_{k} \in H^{-1 / 2}\left(\sigma_{k}\right)$ such that

$$
\left\langle\tilde{\mu}_{k} \mid w\right\rangle_{H^{1 / 2}\left(\sigma_{k}\right)}=\left\langle\mu \mid E_{\left[\sigma_{k}, \partial \Omega\right]}(w)\right\rangle_{H^{1 / 2}(\partial \Omega)},
$$


for any $w \in H^{1 / 2}\left(\sigma_{k}\right)$. We can thus introduce an operator $\Lambda_{\left[\partial \Omega, \sigma_{k}\right]}: H^{-1 / 2}(\partial \Omega) \longrightarrow H^{-1 / 2}\left(\sigma_{k}\right)$ and make $\Lambda_{\left[\partial \Omega, \sigma_{k}\right]}(\mu) \stackrel{\text { def }}{=} \tilde{\mu}_{k}$. The result in (9b) then follows.

Proof (3). Let $\mu \in H^{-1 / 2}(\partial \Omega)$ and $g \in H^{1 / 2}(\partial \Omega)$ be arbitrary. For each $k=1, \cdots, c,\left.g\right|_{\sigma_{k}} \in H^{1 / 2}\left(\sigma_{k}\right)$ (see Property 2 in Appendix B). Therefore, for each $k$, we have $E_{\left[\sigma_{k}, \partial \Omega\right]}\left(\left.g\right|_{\sigma_{k}}\right) \in H^{1 / 2}(\partial \Omega)$ (see Conclusion 1 of this theorem). This motivates our writing

$$
g=\sum_{k=1}^{c} E_{\left[\sigma_{k}, \partial \Omega\right]}\left(\left.g\right|_{\sigma_{k}}\right)
$$

so that

$$
\langle\mu \mid g\rangle_{H^{1 / 2}(\partial \Omega)}=\left\langle\mu \mid \sum_{k=1}^{c} E_{\left[\sigma_{k}, \partial \Omega\right]}\left(\left.g\right|_{\sigma_{k}}\right)\right\rangle_{H^{1 / 2}(\partial \Omega)}=\sum_{k=1}^{c}\left\langle\Lambda_{\left[\partial \Omega, \sigma_{k}\right]}(\mu)|g|_{\sigma_{k}}\right\rangle_{H^{1 / 2}\left(\sigma_{k}\right)},
$$

due to the linearity of $\mu$ and to (9b).

\section{APPENDIX D. THE THEOREM 7.1}

Since $\Sigma$ is a simple closed curve in $\mathbb{R}^{2}$ (see Fig. A1), its complement $\mathbb{R}^{2} \backslash \Sigma$ is comprised of two connected components, the interior (denoted by $\Sigma^{o}$ ) and the exterior (denoted by ext $\Sigma$ ), such that $\Sigma^{o}$ is bounded and $\partial \Sigma^{o}=\partial($ ext $\Sigma)=\Sigma$ (see Jordan Curve theorem [49]). Let $\theta$ be an arbitrary nonzero element of $H^{-1 / 2}(\Sigma)$. Since $H^{1 / 2}\left(\partial \Sigma^{o}\right)$ is a Hilbert space (Property 1, Appendix B), Riesz's Representation theorem [42] asserts the existence of an operator $\mathcal{R}: H^{-1 / 2}\left(\partial \Sigma^{o}\right) \longrightarrow H^{1 / 2}\left(\partial \Sigma^{o}\right)$ such that

$$
\langle\theta \mid t\rangle_{H^{1 / 2}\left(\partial \Sigma^{o}\right)}=(t, \mathcal{R}(\theta))_{H^{1 / 2}\left(\partial \Sigma^{o}\right)^{\prime}}
$$

for any $t \in H^{1 / 2}\left(\partial \Sigma^{o}\right)$, and

$$
\|\theta\|_{H^{-1 / 2}\left(\partial \Sigma^{o}\right)}=\|\mathcal{R}(\theta)\|_{H^{1 / 2}\left(\partial \Sigma^{o}\right)},
$$

where $(\cdot, \cdot)_{H^{1 / 2}\left(\partial \Sigma^{o}\right)}$ denotes the inner product in the space $H^{1 / 2}\left(\partial \Sigma^{o}\right)$. The spaces $H^{1}\left(\Sigma^{o}\right)$ and $H^{1 / 2}\left(\partial \Sigma^{o}\right)$ are Hilbert spaces. The trace operator $\gamma_{\partial \Sigma^{o}}: H^{1}\left(\Sigma^{o}\right) \longrightarrow H^{1 / 2}\left(\partial \Sigma^{o}\right)$ is continuous (Theorem A.1), and therefore it admits an adjoint operator $\gamma_{\partial \Sigma^{o}}^{T}: H^{1 / 2}\left(\partial \Sigma^{o}\right) \longrightarrow H^{1}\left(\Sigma^{o}\right)$ defined as [42]

$$
\left(\gamma_{\partial \Sigma^{o}}(w), g\right)_{H^{1 / 2}\left(\partial \Sigma^{o}\right)}=\left(w, \gamma_{\partial \Sigma^{o}}^{T}(g)\right)_{H^{1}\left(\Sigma^{o}\right)^{\prime}}
$$

for any $(w, g) \in H^{1}\left(\Sigma^{o}\right) \times H^{1 / 2}\left(\partial \Sigma^{o}\right)$, where $(\cdot, \cdot)_{H^{1}\left(\Sigma^{o}\right)}$ denotes the inner product in the space $H^{1}\left(\Sigma^{o}\right)$.

Let $w \in H^{1}\left(\Sigma^{o}\right)$ be arbitrary. Since the trace $\gamma_{\partial \Sigma^{o}}(w)$ belongs to $H^{1 / 2}\left(\partial \Sigma^{o}\right)$, we make $t=\gamma_{\partial \Sigma^{o}}(w)$ in (D.1) and conclude that

$$
\left\langle\theta \mid \gamma_{\partial \Sigma^{o}}(w)\right\rangle_{H^{1 / 2}\left(\partial \Sigma^{o}\right)}=\left(\gamma_{\partial \Sigma^{o}}(w), \mathcal{R}(\theta)\right)_{H^{1 / 2}\left(\partial \Sigma^{o}\right)},
$$

for any $w \in H^{1}\left(\Sigma^{o}\right)$. We now make $g=\mathcal{R}(\theta)$ in (D.3) and conclude that

$$
\left(\gamma \partial \Sigma^{o}(w), \mathcal{R}(\theta)\right)_{H^{1 / 2}\left(\partial \Sigma^{o}\right)}=\left(w, \gamma_{\partial \Sigma^{o}}^{T}(\mathcal{R}(\theta))\right)_{H^{1}\left(\Sigma^{o}\right)},
$$

for any $w \in H^{1}\left(\Sigma^{o}\right)$.

Let $\gamma_{\partial \Sigma^{o}}^{-}: H^{1 / 2}\left(\partial \Sigma^{o}\right) \longrightarrow H^{1}\left(\Sigma^{o}\right)$ be the right inverse trace operator, according to Appendix A. Since $\mathcal{R}(\theta) \in H^{1 / 2}\left(\partial \Sigma^{o}\right)$ (see (D.1)), it follows that $\gamma_{\partial \Sigma^{o}}^{-}(\mathcal{R}(\theta)) \in H^{1}\left(\Sigma^{o}\right)$. We make $w=\gamma_{\partial \Sigma^{o}}^{-}(\mathcal{R}(\theta)$ ) in (D.5)

$$
\left(\gamma_{\partial \Sigma^{o}}\left(\gamma_{\partial \Sigma^{o}}^{-}(\mathcal{R}(\theta))\right), \mathcal{R}(\theta)\right)_{H^{1 / 2}\left(\partial \Sigma^{o}\right)}=\left(\gamma_{\partial \Sigma^{o}}^{-}(\mathcal{R}(\theta)), \gamma_{\partial \Sigma^{o}}^{T}(\mathcal{R}(\theta))\right)_{H^{1}\left(\Sigma^{o}\right)} \cdot
$$

But $\gamma_{\partial \Sigma^{o}}\left(\gamma_{\partial \Sigma^{o}}^{-}(\mathcal{R}(\theta))\right)=\mathcal{R}(\theta)\left(\right.$ make $D=\Sigma^{o}$ and $g=\mathcal{R}(\theta)$ in (A.1)), so that (D.6) becomes

$$
(\mathcal{R}(\theta), \mathcal{R}(\theta))_{H^{1 / 2}\left(\partial \Sigma^{o}\right)}=\left(\gamma_{\partial \Sigma^{o}}^{-}(\mathcal{R}(\theta)), \gamma_{\partial \Sigma^{o}}^{T}(\mathcal{R}(\theta))\right)_{H^{1}\left(\Sigma^{o}\right)^{\prime}}
$$




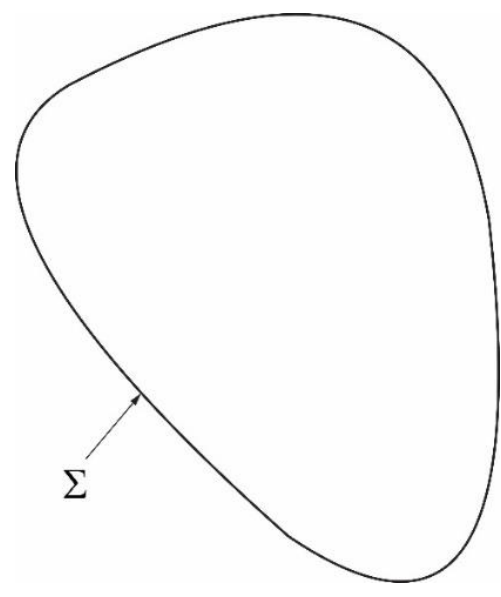

(a)

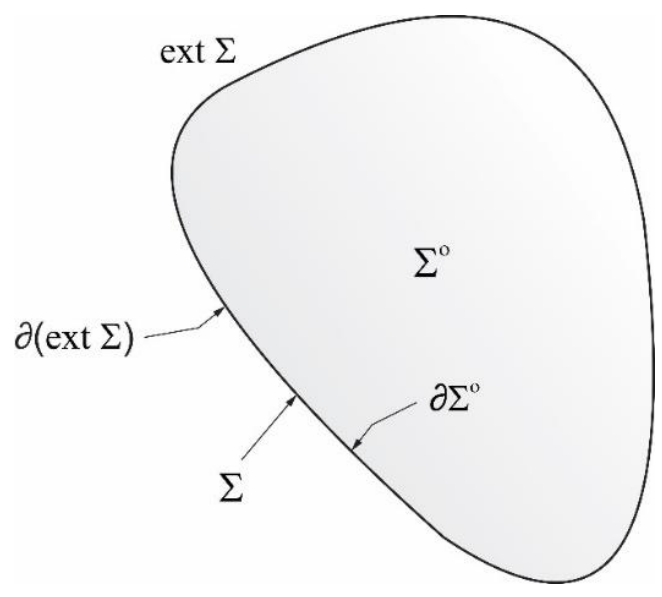

(b)

Fig. A1. The geometrical setting for the application of Theorem 7.1. (a) A simple and closed Lipschitz continuous curve $\Sigma$ in $\mathbb{R}^{2}$. (b) The bounded region $\Sigma^{o}$ corresponds to the interior of curve $\Sigma$, whereas the region ext $\Sigma$ corresponds to the exterior of the curve. It follows that $\partial \Sigma^{0}=\partial(\operatorname{ext} \Sigma)=\Sigma$

or, by the definition of norms in Hilbert spaces [42]

$$
\|\mathcal{R}(\theta)\|_{H^{1 / 2}\left(\partial \Sigma^{o}\right)}^{2}=\left(\gamma_{\partial \Sigma^{o}}^{-}(\mathcal{R}(\theta)), \gamma_{\partial \Sigma^{o}}^{T}(\mathcal{R}(\theta))\right)_{H^{1}\left(\Sigma^{o}\right)} .
$$

The left-hand side of (D.8) is a nonnegative real number, and so is the right-hand side. In this way,

$$
\begin{aligned}
\left(\gamma_{\partial \Sigma^{o}}^{-}(\mathcal{R}(\theta)), \gamma_{\partial \Sigma^{o}}^{T}(\mathcal{R}(\theta))\right)_{H^{1}\left(\Sigma^{o}\right)} & =\left|\left(\gamma_{\partial \Sigma^{o}}^{-}(\mathcal{R}(\theta)), \gamma_{\partial \Sigma^{o}}^{T}(\mathcal{R}(\theta))\right)_{H^{1}\left(\Sigma^{o}\right)}\right| \\
& \leq\left\|\gamma_{\partial \Sigma^{o}}^{-}(\mathcal{R}(\theta))\right\|_{H^{1}\left(\Sigma^{o}\right)}\left\|\gamma_{\partial \Sigma^{o}}^{T}(\mathcal{R}(\theta))\right\|_{H^{1}\left(\Sigma^{o}\right)} \\
& \leq C_{\Sigma^{o}}\|\mathcal{R}(\theta)\|_{H^{1 / 2}\left(\partial \Sigma^{o}\right)}\left\|\gamma_{\partial \Sigma^{o}}^{T}(\mathcal{R}(\theta))\right\|_{H^{1}\left(\Sigma^{o}\right)^{\prime}},
\end{aligned}
$$

where we used Cauchy-Schwarz inequality, and made $D=\Sigma^{o}$ and $g=\mathcal{R}(\theta)$ in (A.2), so that $C_{\Sigma^{o}}$ is a positive constant (depending on the geometry of region $\Sigma^{o}$ ). From (D.8), (D.9), and (D.2), we obtain

$$
\|\theta\|_{H^{-1 / 2}\left(\partial \Sigma^{o}\right)} \leq C_{\Sigma^{o}}\left\|\gamma_{\partial \Sigma^{o}}^{T}(\mathcal{R}(\theta))\right\|_{H^{1}\left(\Sigma^{o}\right)^{\prime}},
$$

since we assumed $\theta$ different from zero. The operators $\mathcal{R}$ (Riesz) and $\gamma_{\partial \Sigma^{\circ}}^{T}$ (adjoint trace) were shown to exist, and so the image of the compound operator $\gamma_{\partial \Sigma^{o}}^{T} \circ \mathcal{R}$ must therefore exist in $H^{1}\left(\Sigma^{o}\right)$. In order to find the element $\left(\gamma_{\partial \Sigma^{o}}^{T} \circ \mathcal{R}\right) \theta=\gamma_{\partial \Sigma^{o}}^{T}(\mathcal{R}(\theta))$, we observe from (D.4) and (D.5) that

$$
\left(w, \gamma_{\partial \Sigma^{o}}^{T}(\mathcal{R}(\theta))\right)_{H^{1}\left(\Sigma^{o}\right)}=\left\langle\theta \mid \gamma_{\partial \Sigma^{o}}(w)\right\rangle_{H^{1 / 2}\left(\partial \Sigma^{o}\right)},
$$

for any $w \in H^{1}\left(\Sigma^{o}\right)$. The unknown $\gamma_{\partial \Sigma^{o}}^{T}(\mathcal{R}(\theta))$ thus satisfies the equality

$$
\int_{\Sigma^{o}}\left[\nabla w \cdot \nabla\left(\gamma_{\partial \Sigma^{o}}^{T}(\mathcal{R}(\theta))\right)^{*}+w\left(\gamma_{\partial \Sigma^{o}}^{T}(\mathcal{R}(\theta))\right)^{*}\right] d \Omega=\left\langle\theta \mid \gamma_{\partial \Sigma^{o}}(w)\right\rangle_{H^{1 / 2}\left(\partial \Sigma^{o}\right)},
$$

for any $w \in H^{1}\left(\Sigma^{o}\right)$, after we expanded the inner product for the (complex-valued) Hilbert space $H^{1}\left(\Sigma^{o}\right)$ in (D.11). If we define $f_{\theta} \stackrel{\text { def }}{=}\left(\gamma_{\partial \Sigma^{o}}^{T}(\mathcal{R}(\theta))\right)^{*}$ and recall that $\partial \Sigma^{o}=\Sigma$ (see Fig. A1), (D.12) becomes

$$
\int_{\Sigma^{o}}\left(\nabla w \cdot \nabla f_{\theta}+w f_{\theta}\right) d \Omega=\left\langle\theta \mid \gamma_{\Sigma}(w)\right\rangle_{H^{1 / 2}(\Sigma)}
$$


for any $w \in H^{1}\left(\Sigma^{o}\right)$. This equality can be interpreted as a problem in weak form; if $\theta$ is given, then we can solve for $f_{\theta}$. After we find $f_{\theta}$, we make

$$
\left\|f_{\theta}\right\|_{H^{1}\left(\Sigma^{o}\right)}=\left\|\left(\gamma_{\partial \Sigma^{o}}^{T}(\mathcal{R}(\theta))\right)^{*}\right\|_{H^{1}\left(\Sigma^{o}\right)}=\left\|\gamma_{\partial \Sigma^{o}}^{T}(\mathcal{R}(\theta))\right\|_{H^{1}\left(\Sigma^{o}\right)^{\prime}},
$$

and the inequality (D.10) becomes

$$
\|\theta\|_{H^{-1 / 2}(\Sigma)} \leq C_{\Sigma^{\mathrm{o}}}\left\|f_{\theta}\right\|_{H^{1}\left(\Sigma^{\mathrm{o}}\right)},
$$

where we again recalled that $\partial \Sigma^{o}=\Sigma$ (see Fig. A1). Since $\theta \in H^{-1 / 2}(\Sigma)$ is arbitrary, and since the constant $C_{\Sigma^{o}}$ does not depend on any particular choice of $\theta$, the conclusions (33a) and (33b) in Theorem 7.1 follow from (D.15) and (D.13), respectively. 